\title{
Espaços regulares com topologias extremas
}

\author{
Antônio de Pádua Franco Filho
}

TESE APRESENTADA AO

INSTITUTO DE MATEMÁTICA E ESTATÍSTICA DA

UNIVERSIDADE DE SÃO PAULO PARA

OBTENÇÃO DO GRAU DE DOUTOR EM

MATEMÁTICA

Área de Concentração: Matemática

Orientador: Profa. Dra. Ofélia Teresa Alas

$\mathrm{Na}$ elaboração desta tese, o autor obteve apoio financeiro parcial da CAPES.

- São Paulo, SP — Dezembro de 2001 - 


\section{Espaços regulares com topologias ex- tremas}

Este exemplar corresponde à redaçao final da tese de doutorado devidamente corrigida e defendida por Antonio de Pádua Franco Filho e aprovada pela comissão julgadora

São Paulo, fevereiro de 2002.

Banca examinadora:

Profa. Dra. Ofelia Teresa Alas (Orientadora) Profa. Dra. Lúcia Renato Junqueira IME-USP

Prof. Dr. Walter A. Carnielli

Prof. Dr. Janey Daccach

IME-USP

Prof. Dr. Francisco A. Doria IFCH-UNICAMP ICMC-USP

UFRJ 


\section{AGRADECIMENTOS}

Aos meus familiares.

A todas as pessoas que com seu trabalho fazem a instituição IME-USP.

A todos alunos e professores que formam o nosso grupo de topologia.

Aos caros amigos Claus, Plínio, Piotr, Lúcia e Ofélia. 
ABSTRACT. We show that the small cardinal number $i=$ $\min \{|\mathcal{A}|: \mathcal{A}$ is a maximal independent family $\}$ has the following topological characterization $i=\min \left\{\kappa \leq c:\{0,1\}^{\kappa}\right.$ has a dense irresolvable countable subspace $\}$, where $\{0,1\}^{\kappa}$ denotes the Cantor cube of weight $\kappa$. As a consequence of this fact, we have in ZFC plus $i=c$ a submaximal countable dense subspace of the Cantor cube of weight $c$. We also have in a model of ZFC plus $i=\aleph_{1}<c$ the same fact.

RESUMO. Vamos mostrar que o pequeno número cardinal $i=\min \{|\mathcal{A}|: \mathcal{A}$ é uma família independente $\}$ tem a seguinte caracterização topológica $i=\min \left\{\kappa \leq c:\{0,1\}^{\kappa}\right.$ tem um subespaço denso enumerável e irresolúvel $\}$, onde $\{0,1\}^{\kappa}$ denota o cubo de Cantor de peso $\kappa$. Como uma conseqüência deste fato, temos em ZFC mais $i=c$ um subespaço denso enumerável submaximal no cubo de Cantor de peso $c$. Vamos mostrar que o mesmo fato vale em um modelo de ZFC mais $i=\aleph_{1}<c$. 


\section{Sumário}

1 Introdução 1

1.1 Apresentação . . . . . . . . . . . . . . . . 1

1.2 Notações e definições . . . . . . . . . . . . . . . 4

2 Espaços maximais $\quad 10$

2.1 Os cardinais $i r r, m \ldots \ldots \ldots$

2.2 O $\pi$-carácter . . . . . . . . . . . . . . . . 19

2.3 pirr . . . . . . . . . . . 26

3 Caracterização do cardinal $i \quad 29$

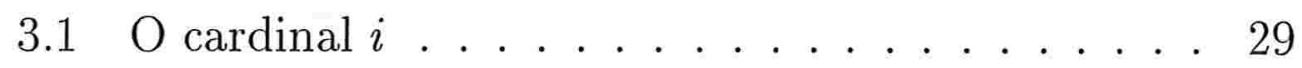

3.2 Espaços Submaximais . . . . . . . . . . . . . . . 34

3.3 Modelo de Bell e Kunen . . . . . . . . . . . . . 40

3.4 Grupos Booleanos enumeráveis . . . . . . . . . 41

4 Espaços homogêneos especiais 49

4.1 Topologias para $A u t(X)$. . . . . . . . . . . . 49

4.2 Métricas completas para $\operatorname{Aut}(X) \ldots . . . . .53$

4.3 Ultramétricas . . . . . . . . . . . . . 56

$4.4(A u t(P), \varrho)$ é homeomorfo a $B(c) \ldots . . . . .57$

4.5 Para espaços uniformes .......... 60 


\section{Capítulo 1}

\section{Introdução}

\subsection{Apresentação}

Vamos considerar algumas questões neste trabalho relativas à existência de espaços regulares enumeráveis com "topologias maximais". Há uma forte relação entre estas topologias e as subálgebras de Boole do conjunto dos subconjuntos dos números naturais $p(\omega)$.

Questão 1.1.1 Em ZFC o cubo de Tychonoff $[0,1]^{c}$ contém um subespaço denso enumerável submaximal?

Esta questão está proposta exatamente nestes termos em [ASTTW], e lá os autores obtiveram em ZFC + BL ( BL significa Lema de Booth, que é a afirmação $p=c$ ) a existência destes espaços. Vamos obter uma resposta em ZFC $+(i=c)$, onde $i$ é o pequeno cardinal relativo às famílias independentes de $p(\omega)$. Como é consistente que $p<i$, este novo resultado é um pouco mais forte que o obtido anteriormente pelos autores de [ASTTW]. 
Questão 1.1.2 Dar uma caracterização topológica para o cardinal $i$.

A proposta acima foi feita pelo professor Koszmider durante um seminário apresentado no IME.

Questão 1.1.3 Obter uma condição suficiente para imergir densamente um espaço regular e enumerável e (mais algumas condições que temos que descobrir) no cubo de Cantor de peso $\kappa, \aleph_{1} \leq \kappa \leq c$.

Questão 1.1.4 Seja $X$ um espaço regular, enumerável, de peso $w(X)=\aleph_{1}$ e de pi-carácter local $\pi \chi(x, X)=\aleph_{1}$ para todo $x \in$ $X$. Se $\beta X$ denota o compactificado de Čech-Stone do espaço $X$, então $\pi \chi(p, \beta X)=\aleph_{1}$ para todo $p \in \beta X$ ?

Vamos obter que os grupos topológicos enumeráveis booleanos cujas topologias são subconjuntos da topologia de Bohr para estes grupos, são densamente imersíveis nos cubos de Cantor de mesmo peso que o peso do grupo. Esta é a melhor resposta que nós obtivemos para a questão1.1.3. A questão 1.1.4 tem resposta afirmativa. Ao respondê-la temos uma prova de que $i \geq \aleph_{1}$, que difere de um simples exercício, uma vez que as construções envolvidas na sua demonstração foram feitas dentro da topologia do espaço em questão e não em $p(\omega)$.

Questão 1.1.5 Existe um espaço regular e enumerável $X$, de peso $w(X)=\aleph_{1}$ e pi-carácter local $\pi \chi(x, X)=\aleph_{1}$ para todo $x \in X$, que não pode ser densamente imerso no cubo de Cantor $\{0,1\}^{\aleph_{1}}$ ? 
$\mathrm{Em} \mathrm{ZFC} \mathrm{+} \mathrm{CH} \mathrm{temos} \mathrm{um} \mathrm{exemplo} \mathrm{que} \mathrm{responde} \mathrm{esta} \mathrm{per-}$ gunta. Veremos no estudo dos espaços regulares enumeráveis de menor peso possível, e que ainda são irresolúveis, que o correspondente cardinal irr que definiremos; satisfará $p \leq i r r \leq i$.

Um outro cardinal $m$ será definido, este estará ligado aos espaços maximais, e satisfará $i r r \leq m$. Entretanto, somente o cardinal irr não terá uma definição nos termos das álgebras de Boole.

A conjectura mais natural é: $i r r=i$ ?

Todavia sua resposta depende de ser estabelecida uma condição suficiente para imersões densa em cubos de Cantor.

Algumas vezes chamamos os cardinais menores ou iguais ao continuum de pequenos cardinais.

Faremos ainda um estudo a respeito do conjunto dos autohomeomorfismos dentro da classe dos espaços completamente metrizáveis, zero-dimensionais, homogêneos $X$ tais que $X^{\omega}$ é homeomorfo ao própio $X$. Neste estudo veremos uma caracterização destes entes envolvidos. Em seguida estudamos uma generalização, para os casos onde tais espaços não são metrizáveis, nos termos de espaços uniformes. O cubo de Cantor $2^{\kappa}$ para um cardinal infinito $\kappa \geq \aleph_{0}$ é um exemplo de tais espaços que não é metrizável se $\kappa>\aleph_{0}$.

Buscamos estabelecer alguns resultados que nos dessem uma resposta de como seus subespaços densos influiam nas topologias que é usual impor ao conjunto dos seus automorfismos. 


\subsection{Notações e definições}

Vamos relembrar as definições de algumas das funções cardinais que usaremos com muita frequência neste texto.

Definição 1.2.1 (Juh) Seja $(X, \tau)$ um espaço topológico.

$O$ peso do espaço $X$ é o menor cardinal infinito $\kappa$ tal que existe uma base de subconjuntos abertos em $X$ com cardinalidade $\leq \kappa$. Notação $w(X)$.

Uma pi-base para um espaço $X$ é uma família $\mathcal{A} \subset \tau \backslash \emptyset$, tal que para todo aberto não vazio $V \in \tau$ existe $N \in \mathcal{A}$ tal que $N \subset V$. O pi-peso é: $\pi w(X)=\min \{|\mathcal{A}|: \mathcal{A}$ é pi-base de $X\}+\aleph_{0}$.

$O$ carácter de um ponto $x \in X$ no espaço $X$ é o menor cardinal infinito $\kappa$ tal que existe uma base local $\mathcal{B} \subset \tau \backslash\{\emptyset\}$, isto é; $\forall V(x \in V \in \tau)$, existe $G \in \mathcal{B}$ tal que $x \in G \subset V$, com cardinalidade $\leq \kappa$. Notação $\chi(x, X)$.

$O$ pi-carácter local de um ponto $x \in X$ no espaço $X$ é o menor cardinal infinito $\kappa$ tal que existe uma pi-base local $\pi \mathcal{B} \subset \tau \backslash\{\emptyset\}$, isto é; $\forall V(x \in V \in \tau)$, existe $G \in \pi \mathcal{B}$ tal que $G \subset V$, com cardinalidade $\leq \kappa$. Notação $\pi \chi(x, X)$.

$O$ "extent" de um espaço $X$, é o menor cardinal infinito $\kappa$ tal que, se $F \subset X$ é discreto e fechado, então $|F| \leq \kappa$. Notação $e(X)$. Notamos que no caso em que $X$ é um espaço compacto, os fechados discretos são finitos, mas o "extent" é infinito.

Um "network" para um espaço $X$ é uma família $\mathcal{N} \subset p(X) \backslash$ $\emptyset$, tal que para todo aberto não vazio $V \in \tau$ e todo ponto $x \in V$ existe $N \in \mathcal{N}$ tal que $x \in N \subset V$.

Dizemos que $\mathcal{C} \subset \tau \backslash\{\emptyset\}$ é uma família celular se seus elementos são subconjuntos abertos dois a dois disjuntos. Analo- 
gamente, definimos celularidade em outras famílias de $p(X)$ tal como nos networks.

$A$ celularidade de um espaço $X$ é: $c(X)=\sup \{|\mathcal{A}|: \mathcal{A} \subset$ $\tau \backslash\{\emptyset\}$ é uma subfamília celular $\}+\aleph_{0}$. Analogamente, seja $\mathcal{N}$ um network, ou um pi-network, definimos: $n c(X)=\sup \{|\mathcal{A}|$ : $\mathcal{A} \subset \mathcal{N}$ é uma subfamília celular $\}+\aleph_{0}$. Analogamente para $\pi$ network: $\pi n c(X)=\sup \{|\mathcal{A}|: \mathcal{A} \subset \mathcal{N}$ é uma subfamilia celular \}$+\aleph_{0}$.

Vamos definir exatamente o que chamaremos de álgebra de Boole de $p(\omega)$.

Definição 1.2.2 (Kun) Uma família $\mathcal{A} \subset p(\omega)$ será chamada de álgebra de Boole (ou de álgebra booleana) se ela satisfaz as seguintes condições:

(1) $\emptyset, \omega \in \mathcal{A}$,

(2) $U, V \in \mathcal{A} \Longrightarrow U \cup V, U \cap V, U \backslash V \in \mathcal{A}$.

Dizemos que $G \in \mathcal{A}$ é um átomo de $\mathcal{A}$ se $V \in \mathcal{A}$ é tal que $V \cap G \neq \emptyset$ então $G \subset V$. Em outros termos, $G$ não pode ser quebrado dentro de $\mathcal{A}$ em dois elementos próprios de $\mathcal{A}$. Dizemos que $\mathcal{A}$ separa pontos de $\omega$ se $\forall x, y \in \omega, x \neq y, \exists V \in \mathcal{A}$ tal que $x \in V$ e $y \in \omega \backslash V$. Em outros termos: $\cap\{V \in \mathcal{A}: x \in$ $V\}=\{x\}$ para cada $x \in \omega$. Assim se $\tau_{\mathcal{A}}$ é a topologia cuja base de abertos é $\mathcal{A}$, então $X=\left(\omega, \tau_{\mathcal{A}}\right)$ é um espaço regular. $\{x\}$ é um atomo de $\mathcal{A}$ se e somente se $\{x\} \in \mathcal{A}$, se e somente se $\{x\} \in \tau_{\mathcal{A}}$, portanto $x$ é um ponto isolado em $X$.

Seja $\mathcal{T} \subset p(\omega)$ uma família não vazia. A álgebra finitamente gerada por esta família, denotamos por $[\mathcal{T}]$, é definida por indução finita como segue:

$[\mathcal{T}]=\cup\left\{\mathcal{V}_{n}: n \in \omega\right\}$ onde : 
(i) $\mathcal{V}_{0}=\{\emptyset, \omega\} \cup\{\omega \backslash T: T \in \mathcal{T}\} \cup \mathcal{T}$.

(ii) $\mathcal{V}_{n+1}=\left\{S \backslash T, S \cap T, S \cup T: S, T \in \mathcal{V}_{n}\right\}$.

Vamos recordar as definições de espaços zero-dimensionais, nas tres versões mais comuns,

$$
\operatorname{ind}(X)=0, \operatorname{dim}(X)=0, \operatorname{Ind}(X)=0 .
$$

Seja $X$ um espaço de Tychonoff.

1.2.3 (Eng) (1) Dizemos que a pequena dimensão indutiva do espaço $X$ é zero, e escrevemos ind $(X)=0$, se dados um ponto $x \in X$ e um subconjunto fechado, $F \subset X$, ao qual o ponto não pertence, existem subconjuntos abertos-fechados $U, V \subset X$ tais que:

$x \in U, F \subset V, U \cap V=\emptyset, U \cup V=X$.

É equivalente dizer que ind $(X)=0$ se e somente se todo ponto $x$ de $X$ admite um sistema fundamental de vizinhanças abertas-fechadas.

(2) Dizemos que a dimensão por recobrimento do espaço $X$ é zero, e escrevemos $\operatorname{dim}(X)=0$, se todo recobrimento formado por dois subconjuntos funcionalmente abertos $\{A, B\}$ de $X$, admite um refinamento formado por dois subconjuntos funcionalmente abertos e disjuntos $\{U, V\}$ que recobre o espaço $X$.

(3) Dizemos que a grande dimensão indutiva de um espaço normal $X$ é zero, e escrevemos Ind $(X)=0$, se dados dois subconjuntos fechados disjuntos, $F, G \subset X$, existem subconjuntos abertos-fechados $U, V \subset X$ tais que:

$F \subset U, G \subset V, U \cap V=\emptyset, U \cup V=X$.

Temos o seguinte fato. 
Fato 1.2.4 (Eng) Seja $X$ é um espaço métrico separável. Então

$$
\begin{aligned}
\operatorname{ind}(X)=0 & \Longleftrightarrow \operatorname{dim}(X)=0 \\
& \Longleftrightarrow \operatorname{Ind}(X)=0
\end{aligned}
$$

1.2.5 (Kun) Dados dois conjuntos $E, A$ usamos a notação: $F n(E, A)=\left\{p: p\right.$ é função e $\operatorname{dom}(p) \in[E]^{<\omega}$ e im $\left.(p) \subset A\right\}$. $[E]^{<\omega}$ para indicar os subconjuntos finitos do conjunto $E$.

Definição 1.2.6 (Co) Sejam $G$ um grupo abeliano, e $T=\{z \in$ $\mathbb{C}:\|z\|=1\}$, o subgrupo multiplicativo de $\mathbb{C}$. Seja hom $(G, T)$ o conjunto de todos os homomorfismos do grupo $G$ no grupo T. A topologia de Bohr do grupo $G$, denotamos por $\tau_{B o h r}$, é a topologia cuja subbase, ou conjunto gerador, é dada por $\left\{h^{-1}(V): V \in \tau(T), h \in h o m(G, T)\right\}$. Em outros termos, seja $\psi: G \longrightarrow G^{\text {hom }(G, T)}$ definida por $\psi(x)(h)=h(x)$. Dê ao grupo $G$ a topologia induzida pela função $\psi$ de $G$ sobre o subespaço $\psi[G]$ do espaço produto $G^{\text {hom }(G, T)}$.

Vamos fazer algumas observações, cuja finalidade é destacar as notações que serão usadas. Dados dois conjuntos $A$ e $X$ denotamos por ${ }^{A} X=\{f: f$ é função e $\operatorname{dom}(f)=A, i m(f) \subset$ $X\}$. Isto é, o conjunto das funções de $A$ em $X$. Definimos para cada $a \in A$ a projeção $\pi_{a}:{ }^{A} X \longrightarrow X$ tal que $\pi_{a}(f)=f(a)$. A topologia produto $\tau_{\text {prod }}$ tem por conjunto gerador ou subbase a família $\left\{\pi_{a}^{-1}(V): V \in \tau\right.$ e $\left.a \in A\right\}$. O espaço topológico produto denotamos por $X^{A}=\left({ }^{A} X, \tau_{\text {prod }}\right)$.

Usamos com frequência as notações: 
$c l_{X}(A)$ e $c l_{\mathcal{B}}(A)$ para indicar a aderência em relação a um espaço $X$ ou em relação a uma álgebra $\mathcal{B}$ e

$\operatorname{int}_{X}(A)$ e $\operatorname{int}_{\mathcal{B}}(A)$ para indicar o interior em relação a um espaço $X$ ou em relação a uma álgebra $\mathcal{B}$.

$f \circ g$ indica a composição de funções e $f\lceil$ a restriçãode funções.

Definição 1.2.7 Seja $(X, \tau)$ um espaço topológico. Dizemos que o espaço $X$ é homogêneo, se dados dois pontos $x, y \in X$ existe um homeomorfismo $h: X \longrightarrow X$ tal que $h(x)=y$.

Vamos a seguir, relembrar alguns resultados, que foram estabelecidos ao longo do século XX. Entre eles dois em particular usamos no capítulo 4.

Fato 1.2.8 (Urysohn e Alexandroff, 1928). Todo espaço topológico completamente metrizável, separável, zerodimensional $X$, que nẫo contém compactos abertos, exceto o vazio, é homeomorfo ao espaço dos números irracionais $P \subset \mathbb{R}$.

Em 1909, Baire mostrou que $P \approx N^{\omega}$, onde $N=\omega$, é o espaço discreto dos números naturais. Seja $\kappa$ um número cardinal infinito. O espaço de Baire de peso $\kappa$, denotamos por $B(\kappa)$, é o produto topológico de $\omega$ cópias do espaço discreto $D(\kappa)$ de cardinalidade $\kappa$. Observamos que $B(\omega)=N^{\omega}$. Dizemos que um espaço $X$ é homogêneo em peso de peso $w(X)=\kappa \geq \aleph_{0}$ se todo aberto não vazio $V \subset X$, como subespaço de $X$ tem peso $w(V)=\kappa$. 
Fato 1.2.9 (A. H. Stone, 1962). Todo espaço topológico completamente metrizável, $X$, com $\operatorname{Ind}(X)=0$, homogêneo em peso e de peso $\kappa \geq \aleph_{1}$, é homeomorfo ao espaço de Baire de peso $\kappa$.

Em 1986, Medvedev,[Med], obteve a caracterização de $B(\kappa) \times$ $\mathbb{Q}$, o que é bem recente; e Sierpinski em 1920, caracterizou topologicamente $\mathbb{Q}$, Brouwer em 1910 caracterizou o conjunto de Cantor. Estes resultados podem ser encontrados, como problema proposto, em [Eng], capítulo 7.

Na medida de nossas necessidades vamos, oportunamente, definindo as noções que usaremos. Em geral na forma " recordamos que ..." 


\section{Capítulo 2}

\section{Espaços maximais}

\subsection{Os cardinais $i r r, m$}

Em 1943, Hewitt introduziu as noções de espaços maximais, submaximais e irresolúveis. Vamos rever estes conceitos neste estudo, dando ênfase às demonstrações que serão mais simples, por estarem com hipóteses adicionais bem fortes. Também vamos introduzir dois novos pequenos números cardinais, que "traduzem" estas noções nos termos das sub-álgebras de Boole do conjunto das partes de $\omega$.

As idéias desenvolvidas na demonstração, do famoso resultado de Šapirovskii, por Juhász,[Juh]pg63, foram decisiva para muitos dos resultados que nós alcançamos neste capítulo.

Em todo este capítulo a palavra espaço indica um elemento da classe dos espaços regulares enumeráveis densos em si mesmos.

Fato 2.1.1 Todo espaço regular e enumerável $X$ é um espaço normal, e ind $(X)=\operatorname{dim}(X)=0$. 
Vamos denotar por $\mathcal{D}_{0}(X)$ a álgebra de Boole dos abertos fechados do espaço $X$.

Definição 2.1.2 Seja $X$ um espaço. Dizemos que o espaço $X$ é irresolúvel se todo denso $D \subset X$ tem interior não vazio em $X$. (Escrevemos int $\operatorname{Es}_{X}(D) \neq \emptyset$ ). Dizemos que o espaço $X$ é superirresolúvel se todo aberto não vazio $V \subset X$ é, como subespaço de $X$, um espaço irresolúvel.

Ainda dizemos que o espaço $X$ é hereditariamente irresolúvel se todo subespaço denso em si mesmo de $X$ é, ele mesmo, um espaço irresolúvel.

A um par de subconjuntos densos disjuntos $D_{0}, D_{1}$ de um espaço $X$ chamamos de uma resolução de $X$.

As duas proposições que seguem abaixo têm demonstrações do mesmo tipo e relativamente naturais. Usamos que a reunião de famílias celulares maximais, são densas no espaço correspondente.

Proposição 2.1.3 Seja $X$ um espaço irresolúvel. Existem abertos fechados $V \subset X$ que são subespaços super-irresolúveis.

Proposição 2.1.4 Seja $X$ um espaço super-irresolúvel. Existem abertos fechados $V \subset X$ que são subespaços hereditariamente irresolúveis.

Prova. Suponhamos que para cada aberto-fechado não vazio $V \subset X$ existe um subconjunto denso em si mesmo $D(V) \subset V$ que é resolúvel. Seja então $\mathcal{D} \subset\left\{D(V): V \in \mathcal{D}_{0}(X) \backslash\{\emptyset\}\right\}$ uma subfamília celular maximal do $\pi$-network $\{D(V): V \in$ 
$\left.\mathcal{D}_{0}(X) \backslash\{\emptyset\}\right\}$. Para cada $A \in \mathcal{D}$ seja $\left\{A_{0}, A_{1}\right\}$ uma resolução deste $A$, e tomamos assim $G_{i}=\cup\left\{A_{i}: A \in \mathcal{D}\right\}$ para $i=0,1$. Como $\mathcal{D}$ é maximal $G=G_{0} \cup G_{1}$ é denso em $X$ e $\left\{G_{0}, G_{1}\right\}$ é uma resolução de $G$. Absurdo, pois $X$ é um espaço irresolúvel.

Definição 2.1.5 Seja o pequeno número cardinal, irr $=\min \{w(X): X$ é um espaço irresolúvel $\}$.

Relembramos que a palavra espaço significa espaços regulares, enumeráveis densos em si mesmos.

Fato 2.1.6 Seja X um espaço hereditariamente irresolúvel. Se a cofinalidade $\operatorname{cf}($ irr $)>\aleph_{0}$ então o subconjunto $\{x \in X$ : $\pi \chi(x, X) \geq i r r\}$ é denso em $X$.

Prova.Suponhamos, por absurdo, que exista um aberto-fechado não vazio $F \subset X$ que, como subespaço de $X$, este espaço satisfaça: $\forall x \in F(\pi \chi(x, F)<i r r)$. Então podemos obter uma topologia regular $\sigma \subset \tau_{F}$, que tem por base de abertos-fechados uma álgebra que separa pontos, finitamente gerada por uma $\pi$ base de $F$ de cardinalidade $\sup \{\pi \chi(x, F): x \in X\}<i r r$. Assim $(F, \sigma)$ é resolúvel,e então $\left(F, \tau_{F}\right)$ também o é.

Definição 2.1.7 Dizemos que um espaço $X=(\omega, \tau)$ sem pontos isolados, é maximal se não existe uma topologia $\sigma$, estritamente mais fina do que $\tau$ que faz de $Y=(\omega, \sigma)$ um espaço sem pontos isolados.

Dizemos que um espaço $X$ é submaximal se todo subconjunto denso $D \subset X$ é aberto em $X$.

Dizemos que um espaço $X$ é extremamente disconexo se a aderência de todo aberto em $X$ ainda for um aberto em $X$. 
Vamos caracterizar os espaços maximais em termos das álgebras de Boole dos abertos fechados destes espaços. Observamos que se um espaço $X$ for resolúvel, então tomando uma resolução deste espaço, $D_{0}, D_{1}$, podemos refinar a topologia de $X$, declarando cada um dos elementos $D_{0}, D_{1}$ abertos nesta nova topologia. Assim fica evidente que todo espaço maximal é um espaço irresolúvel.

Proposição 2.1.8 Seja $X=(\omega, \tau)$ um espaço. São equivalentes as seguintes afirmações:

(1) o espaço $X$ é maximal;

(2) a sub-algebra $\mathcal{D}_{0}(X) \subset p(\omega)$ é maximal sem átomos.

Prova.(1) $\Longrightarrow(2)$ Suponhamos que $A, B \in p(\omega)$ são tais que $A \cap B=\emptyset$ e $A \cup B=\omega$ e $\left[\mathcal{D}_{0}(X) \cup\{A, B\}\right]=\mathcal{A}$ é uma subálgebra de Boole sem átomos. Seja $\sigma=\tau_{\mathcal{A}}$ a topologia que refina $\tau$. Como $X$ é maximal, e $\sigma$ não tem pontos isolados, temos que $\sigma=\tau, \operatorname{assim} \mathcal{A}=\mathcal{D}_{0}(X)$.

$(2) \Longrightarrow(1)$ Suponhamos que uma topologia regular $\sigma$ refina a topologia $\tau$, seja $Y=(\omega, \sigma)$ o espaço regular obtido. Como $\mathcal{D}_{0}(X) \subset \mathcal{D}_{0}(Y)$ implica que $\mathcal{D}_{0}(X)=\mathcal{D}_{0}(Y)$ segue que $\sigma=\tau$.

Proposição 2.1.9 Se $X=(\omega, \tau)$ é um espaço maximal então todo subconjunto fechado denso em si mesmo $F$ é também aberto.

Prova. Suponhamos que $F$ é um subconjunto fechado denso em si mesmo, e que existe um ponto $x \in F \cap c l_{X}(X \backslash F)$. Então, seja $Y=(\omega, \sigma)$ o espaço cuja topologia tem por subbase a família $\mathcal{D}_{0}(X) \cup\{F, X \backslash F\}$. Como a topologia de $Y$ refina a topologia 
de $X$, se supormos que $X$ é um espaço maximal, então existe um ponto isolado $a \in Y$. Este ponto está necessariamente em $c l_{X}(X \backslash F) \cap F$. Seja $W \subset X$ um aberto-fechado de $X$ tal que $\{a\}=W \cap F$. Assim $a$ é um ponto isolado de $F \subset X$. Absurdo.

Proposição 2.1.10 Seja $X=(\omega, \tau)$ um espaço submaximal. São equivalentes as seguintes afirmações:

(1) o espaço $X$ é maximal

(2) Para todo $x \in X$ e todo par de subconjuntos abertos $A, B \subset X$ tais que $A \cap B=\emptyset$ e $A \cup B=X \backslash\{x\}$ um deles é fechado.

Prova. Suponhamos que existam $x \in X$ e um par de subconjuntos abertos não fechados $A, B \subset X$ tais que $A \cap B=\emptyset \mathrm{e}$ $A \cup B=X \backslash\{x\}$. Então seja $A \cup\{x\}=V$ e definimos a subálgebra $\left[\mathcal{D}_{0}(X) \cup\{V, X \backslash V\}\right]$, que não tem átomos. Assim a negação de (2) implica a negação de (1).

Definição 2.1.11 Seja $\mathcal{A} \subset p(\omega)$ uma álgebra sem átomos.Dizemos que uma sub-álgebra $\mathcal{B} \subset \mathcal{A}$ é uma $\pi$-base de $\mathcal{A}$ se para todo elemento não vazio $V \in \mathcal{A}$ existe $T \in \mathcal{B} \backslash\{\emptyset\}$ tal que $T \subset V$. Seja

$\pi(\mathcal{A})=\min \{|\mathcal{B}|: \mathcal{B}$ é $\pi$-base de $\mathcal{A}\}$.

Seja o pequeno cardinal,

$m=\min \{\pi(X): X$ é um espaço maximal $\}$, onde $\pi(X)=\sup \{\pi \chi(x, X): x \in X\}$.

Proposição 2.1.12 Seja $\mathcal{A}$ uma álgebra de Boole maximal de $\pi$-carácter mínimo. Então $m=\pi(\mathcal{A})$. 
Prova. Pela definição destes números cardinais, usando os fatos estabelecidos acima na proposição 2.1.8, e observando que a noção de $\pi$-base tanto para os espaços topológicos, como para as álgebras de Boole são equivalentes, segue o fato enunciado.

\section{Proposição 2.1.13 irr $\leq m$}

Prova. Por absurdo, suponhamos que $m<i r r$. Seja $X=(\omega, \tau)$ um espaço maximal com $\pi$-base de abertos-fechados $\mathcal{B} \subset \mathcal{D}_{0}(X)$ tal que $|\mathcal{B}|=m<$ irr.

Como o espaço $X$ é enumerável, existe $\mathcal{A} \subset \mathcal{D}_{0}(X)$ tal que para cada $x \in X$ a família $\mathcal{A}(x)=\{A \in \mathcal{A}: x \in A\} \subset \mathcal{A}$ satisfaz:

$\cap \mathcal{A}(x)=\{x\}$ e $|\mathcal{A}(x)|=\omega$.

Seja $\sigma \subset \tau$ a topologia que tem por base de abertos-fechados a álgebra de Boole finitamente gerada por $\mathcal{V}=\mathcal{B} \cup \mathcal{A}$. O espaço $Y=(\omega, \sigma)$ é resolúvel, porque tem peso $w(Y) \leq|\mathcal{V}|=m<i r r$. Por outro lado, se $D \subset Y$ é denso em $Y$ e $i n t_{Y}(D)=\emptyset$ então para um aberto-fechado não vazio $W \subset X$ temos que $\exists V \in \mathcal{V}$ tal que $\emptyset \neq V \subset W$ e $V \cap D \neq \emptyset$. Assim $D$ é denso em $X$ e portanto tem interior não vazio em $X$. Mas então int $t_{Y}(D) \neq \emptyset$. Absurdo

Lema 2.1.14 Todo espaço regular, enumerável, denso em si mesmo, $X=(\omega, \tau)$, que tem pi-peso $\pi w(X)=\aleph_{0}$, é um espaço resolúvel.

Prova. Como na demonstração da proposição 2.1.13, podemos escolher uma família enumerável, $\mathcal{A} \subset \mathcal{D}_{0}(X)$, que separa pontos 
de $X$, e que contém uma pi-base de $X$, e cuja álgebra de Boole finitamente gerada por esta família, $[\mathcal{A}]$, é base de uma topologia $\sigma \subset \tau$, que faz de $Y=(\omega, \sigma)$, uma cópia do espaço dos números racionais $\mathbb{Q}$. Logo $Y$ é resolúvel. Então, como no argumento final da proposição acima, $X$ é um espaço resolúvel.

Construção 2.1.15 Vamos construir uma cadeia crescente de topologias regulares $\left\langle\tau_{\alpha}: \alpha \in \Gamma>\right.$, onde $\Gamma$ é um ordinal limite, de modo que se $\mu$ é uma topologia regular, que satisfaz: $\bigcup_{\alpha \in \Gamma} \tau_{\alpha} \subset \mu$, então o espaço $(\omega, \mu)$ tem um ponto isolado.

Fixamos $X_{0}=\left(\omega, \tau_{0}\right)$ uma cópia do espaço dos números racionais, e $\mathcal{B}_{0} \subset \mathcal{D}_{0}\left(X_{0}\right)$ uma base enumerável de $X_{0}$. Suponhamos $X_{\alpha}=\left(\omega, \tau_{\alpha}\right)$ para todos $\alpha \in \beta$ já foram definidos, onde $\beta$ é um ordinal limite, e que $\mathcal{B}_{0} \subset \tau_{\alpha}$ é uma pi-base de cada um destes espaços, $X_{\alpha}$. Seja então $\left(\omega, \tau_{\beta}\right)=X_{\beta}$ o espaço regular, cuja topologia tem por base de abertos a família $\cup\left\{\mathcal{D}_{0}\left(X_{\alpha}\right): \alpha \in \beta\right\}$. Há duas possibilidades:

Se $W \subset X_{\beta}$ é um átomo, seja $\Gamma=\beta$, e terminamos.

Se $W \subset X_{\beta}$ é um aberto-fechado infinito, existe $\alpha \in \beta$ e $\emptyset \neq V \in \mathcal{D}_{0}\left(X_{\alpha}\right)$ tal que $V \subset W$, portanto existe $B \in \mathcal{B}_{0}$ tal que $B \subset V \subset W$, o que nos mostra que $\mathcal{B}_{0}$ é pi-base de $X_{\beta}$.

Vamos supor que $X_{\alpha}$ está definido, satisfaz todas as condições pedidas, não tem pontos isolados. Podemos tomar um subconjunto aberto não fechado $A \subset X_{\alpha}$, tal que $X_{\alpha} \backslash c_{X_{\alpha}}(A) \neq \emptyset$.

Seja então, a topologia $\tau_{\alpha+1}$, cuja base de abertos é a álgebra finitamente gerada por $\mathcal{D}_{0}\left(X_{\alpha}\right) \cup\{A, X \backslash A\}$. Então $X_{\alpha+1}=$ $\left(\omega, \tau_{\alpha+1}\right)$ é um espaço regular, sem pontos isolados, e que tem $\mathcal{B}_{0}$ por pi-base. Portanto não é um espaço maximal, pelo lema 2.1.14.

Assim a indução transfinita termina quando para um ordinal limite $\Gamma$ o espaço $X_{\Gamma}$, tem um ponto isolado. 
O exemplo acima serve para mostrar que a construção de espaços maximais depende de alguma condição forte, como iniciar o procedimento com um espaço irresolúvel, e ainda essegurar que nos passos limites não ocorram pontos isolados. O fato logo abaixo enunciado, é bem conhecido, mas o procedimento usado na demonstração, não é comum, pois em geral, as construções de exemplos de espaços maximais, são feitas por indução transfinita. Daí, o nosso interesse.

Fato 2.1.16 Existem espaços regulares enumeráveis densos em si mesmos que são espaços maximais.

Prova. Seja $\operatorname{Alg}(\omega)$ a família de todas as sub-álgebras de $p(\omega)$ ordenadas pela inclusão, que satisfazem as seguintes condições: $\forall \mathcal{A} \in \operatorname{Alg}(\omega)$ temos

$(1) \emptyset \neq V \in \mathcal{A}$ implica $|V|=\omega$.

(2) $\forall n \in \omega, \cap\{V \in \mathcal{A}: n \in V\}=\{n\}$.

Se $\mathcal{D} \subset A l g(\omega)$ é uma subfamília não vazia totalmente ordenada pela inclusão, então a sub-álgebra $\cup \mathcal{D}$ ainda satisfaz (1) e (2), portanto $A l g(\omega)$ é ordenada indutiva. Pelo lema de Kuratowski-Zorn existem $\mathcal{A} \in A l g(\omega)$ que são maximais. Seja $X=\left(\omega, \tau_{\mathcal{A}}\right)$ o espaço cuja topologia tem por base de abertos uma álgebra maximal $\mathcal{A}$. Por (1) $X$ é denso em si mesmo, e por (2) $X$ é um espaço regular. Da proposição $2.1 .8, X$ é um espaço maximal.

A pedido da banca examinadora dessa tese, vamos acrescentar uma demonstração do fato acima por indução transfinita.

Vamos construir uma cadeia crescente de topologias regulares $<\tau_{\alpha}: \alpha \in \Gamma>$, onde $\Gamma$ é um ordinal limite, de modo que se 
$\mu$ é uma topologia regular, que satisfaz: $\bigcup_{\alpha \in \Gamma} \tau_{\alpha} \subset \mu$, então o espaço $(\omega, \mu)$ é maximal.

Fixamos um espaço hereditariamente irresolúvel $X_{0}=\left(\omega, \tau_{0}\right)$ e $\mathcal{B}_{0} \subset \mathcal{D}_{0}\left(X_{0}\right)$ uma base desse espaço $X_{0}$. Ainda pedimos que todo subconjunto desse espaço cujo interior é o vazio tenha um ponto isolado.

Suponhamos $X_{\alpha}=\left(\omega, \tau_{\alpha}\right)$ para todos $\alpha \in \beta$ já foram definidos, onde $\beta$ é um ordinal limite, e que $\mathcal{B}_{0} \subset \tau_{\alpha}$ é uma pi-base de cada um destes espaços, $X_{\alpha}$. Seja então $\left(\omega, \tau_{\beta}\right)=X_{\beta}$ o espaço regular, cuja topologia tem por base de abertos a família $\cup\left\{\mathcal{D}_{0}\left(X_{\alpha}\right): \alpha \in \beta\right\}$.

Se $W \subset X_{\beta}$ é um aberto-fechado (infinito), existe $\alpha \in \beta$ e $\emptyset \neq V \in \mathcal{D}_{0}\left(X_{\alpha}\right.$ tal que $V \subset W$, portanto, existe $B \in \mathcal{B}_{0}$ tal que $B \subset V \subset W$, o que nos mostra que $\mathcal{B}_{0}$ é pi-base de $X_{\beta}$.

Vamos supor que $X_{\alpha}$ está definido, satisfaz todas as condições pedidas, não tem pontos isolados.

Tomamos um subconjunto aberto regular não fechado $A \subset$ $X_{\alpha}$.

Seja então, a topologia $\tau_{\alpha+1}$, cuja base de abertos é a álgebra finitamente gerada por $\mathcal{D}_{0}\left(X_{\alpha}\right) \cup\{A, X \backslash A\}$. Então $X_{\alpha+1}=$ $\left(\omega, \tau_{\alpha+1}\right)$ é um espaço regular, sem pontos isolados, e que tem $\mathcal{B}_{0}$ por pi-base.

Assim a indução transfinita, para um ordinal limite $\Gamma$ estabelece o espaço $X_{\Gamma}$, denso em si mesmo, de modo que todo aberto regular desse espaço é um aberto-fechado, e todo subconjunto cujo interior é o vazio, tem pontos isolados. Logo $\mathcal{D}_{0}\left(X_{\Gamma}\right)$ é maximal.

Definição 2.1.17 $\lambda=\min \left\{\kappa \leq c: \exists A \subset\{0,1\}^{\kappa}\right.$ subespaço denso enumerável irresolúvel\}. 
Teorema 2.1.18 $\mathrm{irr} \leq \lambda$.

Prova. Pela definição de $\lambda$ seja $A \subset\{0,1\}^{\lambda}$ um subespaço denso enumerável e irresolúvel. Pela definição de $i r r$ temos que $i r r \leq$ $w(A)=\lambda$.

Uma questão natural, que é oportuno colocar aqui, é a seguinte: $i r r=\lambda$ ? Para respondê-la é necessário saber fazer a imersão densa de um espaço regular e enumerável no cubo de Cantor de peso irr. Não teremos uma resposta a esta questão. Vamos obter mais adiante algumas condições, que nos permitirá mostrar a grande dificuldade que envolve responder a esta pergunta.

\section{$2.2 \mathrm{O} \pi$-carácter}

Fato 2.2.1 (Eng, cap7) Seja $(X, \tau)$ um espaço de Tychonof. Denotamos o compactificado de Čech-Stone deste espaço por $\beta X$ como é usual. Temos:

(1) $\operatorname{dim}(X)=0 \Longleftrightarrow \operatorname{dim}(\beta X)=0$.

Podemos, como consequência de (1), considerar $\beta X$ como o conjunto de todos os filtros maximais $p \in \mathcal{D}_{0}(X)$, com a topologia cuja a base de abertos-fechados é dada pela família $\left\{W^{*}\right.$ : $\left.W \in \mathcal{D}_{0}(X)\right\}$, onde, por definição, $W^{*}=\{p \in \beta X: W \in p\}, e$ assim, $c_{\beta X}(W)=W^{*}$.

Vamos fixar um espaço regular enumerável denso em si mesmo $(X, \tau)$ de peso $w(X)=\aleph_{1}$ e com pi-carácter local $\pi \chi(x, X)=$ $\aleph_{1}$ para cada ponto $x \in X$. Uma questão que nos propusemos responder é: vale que $\pi \chi(p, \beta X)=\aleph_{1}$ para todos os pontos de 
$p \in \beta X$ ? Outra destas questões é estabelecer um exemplo de tais espaços $X$ que não é subespaço denso do cubo de Cantor $2^{\aleph_{1}}$.

Proposição 2.2.2 Para todo $p \in \beta X$ temos:

$\pi \chi(p, \beta X) \leq \aleph_{1}$.

Prova. Fixemos uma base de abertos-fechados $\mathcal{B} \subset \mathcal{D}_{0}(X)$ com cardinalidade $|\mathcal{B}|=\aleph_{1}$. Dado um ponto $p \in \beta X$ e um elemento $V$ deste filtro maximal $p$ existe um elemento $B \in \mathcal{B}$ tal que $B \subset V$ porque $\mathcal{B}$ é base de $X$. Assim $c l_{\beta X}(B) \subset c l_{\beta X}(V)$, portanto, $\left\{\operatorname{cl}_{\beta X}(V): V \in \mathcal{B}\right\}$ é uma pi-base de $\beta X$.

Lema 2.2.3 Seja $\mathcal{A} \subset \mathcal{D}_{0}(X) \backslash\{\emptyset\}$ uma subfamília enumerável. Existem abertos-fechados $V_{0}, V_{1}$ em $X$ tais que $V_{0} \cap V_{1}=\emptyset e$ $V_{0} \cup V_{1}=X$ e para todo $T \in \mathcal{A}$ vale $T \cap V_{0} \neq \emptyset \neq T \cap V_{1}$.

Prova. Por indução finita, vamos construir uma família celular de abertos-fechados $\mathcal{D}$, que recobre o espaço $X$, tal que:

(1) Se $\mathcal{D}$ é finita, então $\mathcal{D}=\left\{U_{0}, U_{1}, \ldots, U_{n}\right\}$ é tal que os abertos-fechados $V_{0}=\bigcup_{j<n} U_{j}$ e $V_{1}=U_{n}$ satisfazem, para todo $T \in \mathcal{A}$ vale que $T \cap V_{0} \neq \emptyset \neq T \cap V_{1}$, ou

(2) se $\mathcal{D}$ é infinita, então $\mathcal{D}=\left\{U_{n}: n \in \omega\right\}$, e para todo $n \in \omega$, vale que $(\forall T \in \mathcal{A})\left(T \not \subset \bigcup_{j \leq n} U_{j}\right)$.

Neste caso (2), dado $T \in \mathcal{D}$ o conjunto $N(T)=\{n \in \omega$ : $\left.U_{n} \cap T \neq \emptyset\right\}$ é infinito. Assim podemos escolher um subconjunto $J=\left\{n_{T}, m_{T}: T \in \mathcal{A}\right\} \subset \omega$ cujos elementos são dois a dois distintos, e tais que $U_{n_{T}} \cap T \neq \emptyset \neq U_{m_{T}} \cap T$. Para tanto, é suficiente enumerar $\mathcal{A}$, na forma, $(\mathcal{A},<) \approx(\omega, \in)$, e por indução finita, escolher $J$. Agora definimos os abertos-fechados:

$$
V_{0}=\bigcup_{T \in \mathcal{A}} U_{n_{T}} \text { e }
$$




$$
\begin{aligned}
& V_{1}=X \backslash V_{0}=\cup\left(\mathcal{D} \backslash\left\{U_{n_{T}}: T \in \mathcal{A}\right\}\right) \\
& \text { e então, por }(2) \text {, para todo } T \in \mathcal{A} \text { vale que: } \\
& V_{0} \cap T \neq \emptyset \neq T \cap \bigcup_{T \in \mathcal{A}} U_{m_{T}} \text { e } \bigcup_{T \in \mathcal{A}} U_{m_{T}} \subset V_{1} .
\end{aligned}
$$

Seja $(X,<)$ uma enumeração do conjunto $X$, na forma de um conjunto bem ordenado do tipo de $(\omega, \epsilon)$.

Por indução em $(X,<)$ vamos escolher uma família de subconjuntos abertos-fechados $\mathcal{D}=\left\{U_{n}: n \in \omega\right\}$, usando a hipótese de que $|\mathcal{A}|=\omega$ e $\pi \chi(x, X)>\omega$ tais que:

(3) $x_{0}=\min _{<}(X) \in U_{0}$ e $\forall T \in \mathcal{A}, T \not \subset U_{0}$

(4) $x_{n+1}=\min _{<}\left(X \backslash \bigcup_{j \leq n} U_{j}\right) \in U_{n+1} \subset X \backslash \bigcup_{j \leq n} U_{j}$ e $\forall T \in$ $\mathcal{A}, T \backslash \bigcup_{j \leq n} U_{j} \not \subset U_{n+1}$.

Se, $\left\{U_{j}^{-}: j \leq n\right\}$, é tal que:

$\bigcup_{j \leq n} U_{j}=W$ e $X \backslash W$

satisfazem as condições pedidas, então terminamos, nossa indução neste passo $n$. Vamos supor que não podemos obter as condições pedidas, num número finito de passos indutivos. Assim, a família obtida $\mathcal{D}=\left\{U_{n}: n \in \omega\right\}$ é celular, e dado $x \in X$ existe $n \in \omega$ tal que $x_{n} \leq x<x_{n+1}$, e então por (4) temos que $x \in \bigcup_{j \leq n} U_{j}$. Logo $\mathcal{D}=\left\{U_{n}: n \in \omega\right\}$, satisfaz (1) e (2).

Proposição 2.2.4 Para todo $p \in \beta X$ temos:

$$
\pi \chi(p, \beta X)=\aleph_{1} .
$$

Prova. Por absurdo, suponhamos que $p \in \beta X$, é um ponto cujo pi-carácter é enumerável. Seja $\mathcal{A}$ uma família de abertosfechados não vazios, em $X$, tal que $(\forall W \in p)(\exists T \in \mathcal{A})$ tal que $T \subset W$. Em outros termos, $\mathcal{A}^{*}=\left\{T^{*}: T \in \mathcal{A}\right\}$, é pi-base local deste ponto $p$ de $\beta X$. Do lema 2.2 .3 podemos tomar um par de abertos-fechados, não vazios, $V_{0}, V_{1} \subset X$ tais que:

$$
\begin{aligned}
& V_{0} \cap V_{1}=\emptyset \text { e } V_{0} \cup V_{1}=X \\
& (\forall T \in \mathcal{A})\left(T \not \subset V_{0}\right) \text { e }\left(T \not \subset V_{1}\right) .
\end{aligned}
$$


Portanto, $V_{0} \in p$ ou $V_{1} \in p$, mas não existe $T^{*} \in \mathcal{A}^{*}$ que esteja contido em $V_{i}^{*}, i=0,1$. Contradição.

Definição 2.2.5 Seja $\mathcal{A} \subset p(\omega)$ uma sub-álgebra. Dizemos que $T \subset \omega$ é transversal à álgebra $\mathcal{A}$ se para todo $V \in \mathcal{A} \backslash\{\emptyset\}$ vale $V \cap T \neq \emptyset \neq V \backslash T$.

Em outros termos podemos dizer que $T$ cinde todos os elementos de $\mathcal{A}$ que não seja o vazio.

Acabamos de ver que se $\pi \chi(x, X)=\aleph_{1}, \forall x \in X$, e se $|\mathcal{A}|=\omega$ então existem elementos $T \in \mathcal{D}_{0}(X)$ transversais à $\mathcal{A}$.

Proposição 2.2.6 Fixemos um espaço regular e enumerável $X=$ $(\omega, \tau)$ com peso $w(X)=\aleph_{1}$ e pi-carácter local $\pi \chi(x, X)=\aleph_{1}$ para todo $x \in X$, e uma sub-álgebra enumerável $\mathcal{A} \subset \mathcal{D}_{0}(X)$, que separa pontos de $\omega$. Seja $Y=\left(\omega, \tau_{\mathcal{A}}\right)$. São equivalentes as seguintes afirmações:

(1) dado $\emptyset \neq W \in \mathcal{D}_{0}(X)$ tal que $\operatorname{int}_{\mathcal{A}}(W)=\emptyset$ existe $T \in$ $\mathcal{D}_{0}(X)$ transversal à $\mathcal{A}$ e $V \in \mathcal{A}$ tal que $T \cap V=W$,

(2) $\pi: \beta X \longrightarrow \beta Y$ é aberta, onde $\pi$ é a única extensão continua da identidade de $X$ sobre $Y$.

Prova.(1) $\Longrightarrow$ (2). Dado um aberto $A \subset \beta X$ e um ponto $p \in A$ podemos tomar um aberto-fechado $W \in p$ tal que $i n t_{\mathcal{A}}(W)=\emptyset$, e $p \in W^{*} \subset A$. Por hipótese existe $T \in \mathcal{D}_{0}(X)$ transversal à $\mathcal{A}$ e $V \in \mathcal{A}$ tal que $T \cap V=W$, e assim, $p \cap \mathcal{D}_{0}(Y)=\pi(p)$ é tal que

$\pi(p) \in \pi\left[W^{*}\right]=\pi\left[T^{*} \cap V^{*}\right]=\pi\left[V^{*}\right]=c l_{\beta Y}(V) \subset \pi[A]$.

Logo a função $\pi$ é aberta.

$(2) \Longrightarrow(1)$. Dado um aberto-fechado não vazio $W \subset X$ tal que, qualquer que seja $V \in \mathcal{A}, V \not \subset W$, no aberto-fechado $\beta X \backslash$ 
$\pi^{-1}\left(\pi\left[W^{*}\right]\right)$, podemos tomar um aberto-fechado $G \subset X$, tal que para todo $\emptyset \neq V \in \mathcal{A}, V \subset X \backslash \pi^{-1}\left(\pi\left[W^{*}\right]\right.$ vale $V \cap G \neq \emptyset \neq$ $V \backslash G$. Seja $T=W \cup G$, o transversal procurado.

A função $\pi$ que estamos tomando acima, "a única extensão contínua da identidade", pode ser definida em termos da inclusão das respectivas álgebras de Boole. Sejam $\mathcal{A}, \mathcal{B} \operatorname{com} \mathcal{A} \subset \mathcal{B}$ duas álgebras de Boole que separam pontos de $\omega$. Para cada filtro maximal $p \subset \mathcal{B}$ definimos o único filtro maximal $\pi(p)=$ $\mathcal{A} \cap p$. Sejam $\eta_{\mathcal{A}} X$ e $\eta_{\mathcal{B}} Y$, as respectivas extensões dos espaços $X, Y$ cujas álgebras acima são suas respectivas bases de abertos. Então a função $\pi$ é a única extensão contínua da identidade.

Nota 2.2.7 Nas condições acima, vamos supor que $W \in \mathcal{B} \backslash \mathcal{A}$. e que a função $\pi$ é aberta, $\mathcal{B}=\mathcal{D}_{0}(X)$ e $\mathcal{A}=\mathcal{D}_{0}(Y)$. Então temos $V \in \mathcal{A}$ e $W_{0} \in \mathcal{B}$ tais que:

$V \cup W_{0}=W$ e $V \cap W_{0}=\emptyset \operatorname{eint}_{Y}\left(W_{0}\right)=\emptyset$.

Com efeito, usando a hipótese que $\pi$ é aberta, temos o abertofechado de $\beta X$ dado por $G^{*}=\pi^{-1}\left(\pi\left[W^{*}\right]\right) \backslash W^{*}$, e o abertofechado, $W^{*} \cap \pi^{-1}\left(\pi\left[G^{*}\right]\right)=W_{0}^{*}$ é subconjunto de $W^{*}$, Temos $p \in\left(W \backslash W_{0}\right)^{*} \Longleftrightarrow \pi^{-1}(\pi(p)) \subset\left(W \backslash W_{0}\right)^{*} \Longleftrightarrow W \backslash W_{0} \in \mathcal{A}$. Seja $V=W \backslash W_{0}=i n t_{Y}(W)$.

2.2.8 Seja $X$ um subespaço denso e enumerável do cubo $2^{\aleph_{1}}$, e $\Omega \subset X$ um aberto-fechado não vazio do espaço $X$. Para cada $p \in F n\left(\aleph_{1}, 2\right)$ seja $V(p)=\{x \in X: x\lceil\operatorname{dom}(p)=p\}$. Dado $a \in \Omega$ existe $p_{a} \in F n\left(\alpha_{1}, 2\right)$ tal que $a \in V\left(p_{a}\right) \subset \Omega$. Como $X$ é enumerável, e dom $\left(p_{a}\right)$ é finito, cada aberto-fechado de $X$ depende de no máximo uma quantidade enumerável de indices, ou coordenadas, $\alpha \in \aleph_{1}$. 
Seja $\mathcal{B} \subset \mathcal{D}_{0}(X)$ uma sub-álgebra enumerável, e então, para cada $\emptyset \neq T \in \mathcal{B}$ e cada $a \in T$, escolhemos $p_{a}(T)$ tal que $V\left(p_{a}(T)\right) \subset T$, e temos o conjunto enumerável dos indices $I_{\mathcal{B}}=\bigcup_{T \in \mathcal{B}}\left(\bigcup\left\{\operatorname{dom}\left(p_{a}(T)\right): a \in T\right\}\right)$

dos quais $\mathcal{B}$ depende.

Um aberto-fechado básico $W(q) \subset X$, é transversal à $\mathcal{B}$, se e somente se $\operatorname{dom}(q) \cap I_{\mathcal{B}}=\emptyset$. Assim temos que as vizinhanças básicas $W(q)$ em $X$ são do tipo:

$V\left(p_{0}\right) \cap V\left(p_{1}\right)$ onde $p_{0}=q \uparrow\left(\operatorname{dom}(q) \cap I_{\mathcal{B}}\right)$ e $p_{1}=q \uparrow\left(\operatorname{dom}(q) \backslash I_{\mathcal{B}}\right)$.

Desta última observação, se $Y$ é o espaço regular (supomos que $\mathcal{B}$ separa pontos de $X$ ), de suporte $X$ e topologia cuja base de abertos é $\mathcal{B}$, a função $\pi$ de $\beta X$ sobre $\beta Y$, é aberta, pela proposiçẫo 2.2.6.

Exemplo 2.2.9 Fixemos, um espaço regular e enumerável $X=$ $(\omega, \tau)$ com peso $w(X)=\aleph_{1}$ e pi-carácter local $\pi \chi(x, X)=\aleph_{1}$ para todo $x \in X$, e uma sub-álgebra enumerável $\mathcal{A} \subset \mathcal{D}_{0}(X)$, que separa pontos de $X$. Seja $Y=\left(\omega, \tau_{\mathcal{A}}\right)$. Suponhamos que a função $\pi: \beta X \longrightarrow \beta Y$ é aberta, onde $\pi$ é a única extensão contínua da identidade de $X$ sobre $Y$. Como $Y$ é uma cópia do espaço dos números racionais, fixamos um par de abertosfechados não vazios e complementares $A, B \subset Y$ em $Y$ e um par $F, G=Y \backslash F$ onde $F$ é uma cópia fechada de $Y$ com interior vazio em $Y$ Definimos uma função contínua e bijetora $h: Y \longrightarrow Y$, tal que $h[A]=F$ e $h[B]=G$, onde para obtê-la, é suficiente tomar $h$ de modo que sua restrição a $A$ e a $B$ sejam homeomorfismos. Seja $\varphi: \beta X \longrightarrow \beta Y$ a única extensão contínua de $h \circ i d$. Esta função não é aberta. Assim a condição (1) da proposição acima falha exatamente para $A=W$ 
que podemos supor satisfazer as condições pedidas no enunciado da mesma.

Com este exemplo fica claro, que para fazer imersões densas nos cubos de Cantor, iremos precisar de hipóteses bem fortes para os nossos espaços. Vamos obter sucesso no caso dos grupos booleanos enumeráveis, com uma topologia bem específica. Para complementar estas observações o próximo teorema mostra-nos que topologias extremas são decisivas para que a questão1.1.3, tenha alguma resposta.

Teorema 2.2.10 ( $Z F C+C H)$ Existem espaços regulares enumeráveis $X=(\omega, \tau)$ com peso $w(X)=\aleph_{1}$ e pi-carácter local $\pi \chi(x, X)=\aleph_{1}$ para todo $x \in X$, que não podem ser densamente imersíveis no cubo de Cantor $\{0,1\}^{\aleph_{1}}$.

Prova. Em [ASTTW] na proposição 2.1 os autores mostram que um espaço maximal não pode ser subespaço denso de um produto de espaços metrizáveis, e o cubo de Cantor é um destes produtos. Assim é suficiente mostrar que existe um espaço maximal $X=(\omega, \tau)$ uniforme em pi-carácter local. Usando a proposição 2.1.16, seja $Y$ um espaço maximal, e definimos o subconjunto $F=\{y \in Y: \pi \chi(y, Y)=\omega\} \subset Y$. Se int $t_{Y}(F) \neq \emptyset$ então como subespaço de $Y$ o espaço $F$ contém um subconjunto aberto-fechado que é um espaço resolúvel,temos uma contradição com o fato de que espaços maximais são espaços submaximais e então são também espaços superirresolúveis.Logo existe um aberto-fechado $\emptyset \neq X \subset Y \backslash F$, e assim este espaço satisfaz: $\pi \chi(x, X)=\aleph_{1}$ para todo $x \in X$. 


\section{$2.3 p, i r r$}

Vamos estabelecer o fato $p \leq i r r$ onde relembramos a definição do pequeno cardinal $p$.

Definição 2.3.1 Uma família $\mathcal{A} \subset p(\omega)$ tem a propriedade da forte interseção finita, e abreviamos por $\mathcal{A}$ tem p.f.i.f, se toda subfamilia finita não vazia, $\mathcal{A}_{0} \subset \mathcal{A}$ tem interseção infinita,isto é; $\left|\cap \mathcal{A}_{0}\right|=\omega$. Dizemos que um subconjunto infinito $B \subset \omega$ é uma pseudo-interseção infinita da família $\mathcal{A}$ se para todo $V \in \mathcal{A}$ temos que $B$ está quase todo contido em $V$, isto é: $|B \backslash V|<\omega$, e também usamos a notação $B \subset^{*} V$. Definimos

$p=\min \{|\mathcal{A}|: \mathcal{A}$ tem p.f.i.f e não existe pseudo-interseção infinita de $\mathcal{A}\}$

Relembramos que um espaço de Hausdorff $X$ é um espaço de Fréchet se vale que:

$(\forall G \subset X)\left(\forall a \in c l_{X}(G)\right)$, existe uma sequência

$\left\langle x_{n}: n \in \omega>\right.$ de pontos de $G$, que converge para o ponto $a$, escrevemos $x_{n} \longrightarrow a$, ou $\lim x_{n}=a$.

Fato 2.3.2 Todo espaço de Hausdorff, $X=(\omega, \tau)$ de peso $w(X)<$ p, é um espaço de Fréchet.

Para ver que vale o fato acima, notamos que todo ponto $x$ tem um sistema fundamental $\mathcal{V}$ de vizinhanças abertas com p.f.i.p ( se não for um ponto isolado) cuja cardinalidade é menor que p. Se $G \subset X$ e $x \in c_{X}(G)$ então $\{G \cap V: V \in \mathcal{V}\}$ tem $p . f . i . f$, e cardinalidade menor que $p$. Uma pseudo-interseção $B=\left\{x_{n}: n \in \omega\right\}$ desta família, é uma sequência que converge para o ponto $x$. 
Fato 2.3.3 Se $X=(\omega, \tau)$ é um espaço de Fréchet, então o espaço $X$ é resolúvel.

Prova. Primeiro vamos descrever o espaço $X$, como uma reunião de fechados com interior vazio em $X$, e com esta finalidade, definimos o conjunto enumerável seq, que irá indexar as sequências de $X$. Enumeramos $X=\left\{x_{n}: n \in \omega\right\}$.

Se $F \subset X$, é um dos fechados que obtivemos, e na ordem que vamos definir, $x \in F$, é o primeiro ponto a ser considerado na indução, então acrescentamos uma sequência, $\{a(n): n \in \omega\}$ tal que $\lim a_{n}=x$ e $(\forall n \in \omega)\left(a_{n} \in X \backslash F\right)$.

Seja seq o conjunto das sequências finitas de números naturais, onde a sequência vazia $<>\in \operatorname{seq}$ e se $s=<n_{0}, n_{1}, \ldots, n_{k}>\epsilon$ $s e q$ então para todo $m \in \omega$ definimos $s * m=<n_{0}, n_{1}, \ldots, n_{k}, m>\epsilon$ seq, e ainda tomamos como comprimento destas sequêcias $l(<>$ )$=0$ e $\left.l\left(<n_{0}, n_{1}, \ldots, n_{k}\right\rangle\right)=k+1$. Definimos uma ordem $\leq$ em seq, tal que:

$t<s$ se e somente se: $l(t)<l(s)$ ou $l(t)=l(s)$ mas então existe $0 \leq j \leq l(s)-1$ tal que $t(j)<s(j)$, usando a notação $s=<s(0), s(1), \ldots, s(l(s))>$.

Por indução no comprimento destas sequências, definimos uma sequência crescente de subconjuntos fechados $<F_{n}: n \in$ $\omega>$ em $X$, todos com interior vazio em $X$, tais que;

(1) $F_{0}=\left\{x_{<>}\right\}$e $x_{<>}=x_{0}$

(2) $F_{n+1} \backslash F_{n}=\left\{x_{s}: s \in \operatorname{seq}, l(s)=n+1\right\}$ para cada $n \in \omega$, é discreto em $X$.

(3) $x_{s * m} \longrightarrow x_{s}$, e $x_{s * 0}=x_{k}$ onde $k=\min \left\{n \in \omega: x_{n} \in\right.$ $\left.X \backslash\left\{x_{t}: t<s\right\}\right)$.

Assim , $\bigcup_{n \in \omega} F_{n}=X$ e definimos os subconjuntos $D_{0}=$ $\left\{x_{<>}\right\} \cup\left\{x_{s}: s(n)=n(\bmod 2), 1 \leq n \leq l(s)-1\right\}$ e $D_{1}=\left\{x_{s}:\right.$ $s(n)=n+1(\bmod 2), 1 \leq n \leq l(s)-1\}$, é uma resolução de $X$. 
Teorema 2.3.4p $\leq$ irr.

Prova. Com efeito, se supormos que irr $<p$ então não existe espaço $X$ de peso irr que seja irresolúvel. Absurdo.

Para finalizar esta seção vamos relembrar que os espaços maximais são também sub-maximais, e estes por sua vez são hereditariamente irresolúveis.

Fato 2.3.5 Seja $X$ um espaço sub-maximal. Se $F \subset X$ é um subconjunto cujo o interior em $X$ é o vazio, então $F$ é um subconjunto discreto de $X$.

Prova. Como $X \backslash F=G$ é um subconjunto denso em $X$ e $X$ é um espaço sub-maximal, então $G$ é um subconjunto aberto de $X$. Agora, se o fechado $F$ tem um ponto de acumulação $x \in X$ então $G \cup\{x\}$ é um subconjunto denso de $X$, que não é aberto, porque $X \backslash(G \cup\{x\})=F \backslash\{x\}$ não é fechado. Contradição.

Fato 2.3.6 Seja $X$ um espaço sub-maximal. Se todo $F \subset X$ subconjunto fechado em $X$, denso em si mesmo, é aberto em $X$, então $X$ é um espaço maximal.

Prova. Por hipótese, a álgebra dos fechados regulares de $X$, coincide com a álgebra dos abertos-fechados de $X$, e também coincide com a álgebra dos abertos regulares de $X$. Assim $\mathcal{D}_{0}(X)$ é maximal, portanto $X$ é maximal. Também $X$ é extremamente disconexo. 


\section{Capítulo 3}

\section{Caracterização do cardinal $i$}

Este capítulo é dedicado a responder as questões 1.1.1 e 1.1.2. Também estabelecemos uma condição suficiente para imergir um espaço regular num cubo de Cantor. É a resposta "melhor possível". O que veremos é que a questão 1.1.3, está definitivamente amarrada aos grupos booleanos com uma topologia que é um subconjunto da topologia de Bohr. É uma consequência do teorema 3.4.11.

\subsection{O cardinal $i$}

Vamos definir este pequeno número cardinal, que está associado às famílias independentes do conjunto das partes do conjunto dos números naturais, e estas famílias, por sua vez, caracterizam as topologias dos subespaços densos e enumeráveis dos cubos de Cantor.

Definição 3.1.1 (Kun,pag 257) $\mathcal{A} \subset p(\omega)$ é uma família independente se e somente se para todo $n, m \in \omega$ e para toda subfamilia finita de elementos dois a dois distintos $a_{0}, \ldots, a_{n}, b_{0}, \ldots, b_{m}$ de $\mathcal{A}$ temos $\left|a_{0} \cap \ldots \cap a_{n} \cap\left(\omega \backslash b_{0}\right) \cap \ldots \cap\left(\omega \backslash b_{m}\right)\right|=\omega$. 
Vamos supor sempre que $|\mathcal{A}|>\omega$ e dizemos que $\mathcal{A}$ é uma família independente maximal se para todo $x \in p(\omega) \backslash \mathcal{A}, \mathcal{A} \cup\{x\}$ não é uma família independente.

Sempre que for conveniente, vamos usar a abreviação "f.i." para indicar que é uma família independente. É o caso abaixo, para que a definição não fique quebrada em duas linhas!

Definição 3.1.2 Vamos definir o pequeno número cardinal; $i=\min \{|\mathcal{A}|: \mathcal{A} \subset p(\omega)$ é uma "f.i." maximal $\}$.

Queremos estabelecer o seguinte fato, $i=\lambda$. Este cardinal foi definido em 2.1.17. Para este fim, que é a caracterização topológica do cardinal $i$, precisamos mostrar os dois lemas que seguem. O primeiro destes lemas estabelece a função, cuja imagem é um subespaço denso num cubo de Cantor, e usaremos a notação introduzida neste lema, para esta função, em todo o nosso trabalho. O segundo lema é uma forma de recíproca do primeiro.

Lema 3.1.3 Seja $\mathcal{A}$ uma família independente de $p(\omega)$ e $\psi_{\mathcal{A}}$ uma função de $\omega$ em $\{0,1\}^{\mathcal{A}}$ definida por:

$\forall x \in \omega, \forall V \in \mathcal{A}$,

$\psi_{\mathcal{A}}(x)(V)=0$ se $x \in V$ ou

$\psi_{\mathcal{A}}(x)(V)=1$ se $x \in \omega \backslash V$.

Então o subespaço $\psi_{\mathcal{A}}[\omega]=A$ é um subespaço denso do cubo de Cantor $\{0,1\}^{\mathcal{A}}$ de peso $|\mathcal{A}|$.

Prova. Para cada função parcial finita, $p \in F n(\mathcal{A}, 2) \backslash\{\emptyset\}$, definimos 


$$
V(p)=(\bigcap\{V \in \operatorname{dom}(p): p(V)=0\} \cap(\bigcap\{\omega \backslash V:(V \in
$$
$\operatorname{dom}(p))(p(V)=1)\})$.

Pela definição de família independente:

$|V(p)|=\omega$, assim podemos escolher um ponto $x \in V(p)$, e pela definição da função $\psi_{\mathcal{A}}$ temos que:

$\psi_{\mathcal{A}}(x)(V)=p(V)$, para todo $V \in \operatorname{dom}(p)$.

Denotamos por:

$\mathcal{B}=\{W(p): p \in F n(\mathcal{A}, 2) \backslash\{\emptyset\}\}$,

a subbase canônica da topologia do cubo de Cantor, onde os subconjuntos abertos-fechados são dados por:

$W(p)=\left\{s \in 2^{\mathcal{A}}: s \mid \operatorname{dom}(p)=p\right\}$. Então $\psi_{\mathcal{A}}[V(p)]=$ $A \cap W(p)$.

Seja $A$ um subespaço denso enumerável do cubo de Cantor, $\{0,1\}^{\kappa}$ para um cardinal infinito $\kappa \leq c$. Vamos supor que $A=(\omega, \tau)$, e denotamos os abertos-fechados da subbase canônica deste cubo por $W(\alpha, i)=\left\{s \in 2^{\kappa}: s(\alpha)=i\right\}$,para todo $\alpha \in \kappa \mathrm{e}$ $i \in 2$. Definimos a correspondente família de subconjuntos de $\omega$ por: $\mathcal{A}=\{A \cap W(\alpha, 0): \alpha<\kappa\} \subset p(\omega)$.

Lema 3.1.4 Nas condições acima, então

(1) $\mathcal{A}$ é uma família independente.

(2) $\mathcal{A}$ é uma família independente maximal se e somente se $A$ é um subespaço irresolúvel.

Prova. (1).Dados $n, m \in \omega$ e $\mathcal{B}=\left\{U_{1}, \ldots, U_{n}, V_{1}, \ldots, V_{m}\right\}$ um conjunto de elementos dois a dois distintos de $\mathcal{A}$, escolhemos $p \in F n(\mathcal{A}, 2) \equiv F n(\kappa, 2)$ de modo que:

(a) $\operatorname{dom}(p)=\mathcal{B}$

(b) $p\left(U_{j}\right)=0$ e $p\left(V_{k}\right)=1$ para $j=1, \ldots, n$ e $k=1, \ldots, m$.

Uma vez que $A$ é denso em $\{0,1\}^{\kappa}$ temos 
$|W(p) \cap A|=\omega$ e $W(p) \cap A=U_{1} \cap \ldots \cap U_{n} \cap\left(\omega \backslash V_{1}\right) \cap \ldots \cap\left(\omega \backslash V_{m}\right)$ o que mostra a veracidade de (1).

Para provar (2) suponha que existem dois subconjuntos densos $D_{0}, D_{1}$ no espaço $A$ tais que $D_{0} \cup D_{1}=A$ e $D_{0} \cap D_{1}=\emptyset$. Então $D_{0} \notin \mathcal{A}$ e assim $\mathcal{A} \cup\left\{D_{0}\right\}=\mathcal{B}$ seria uma família independente.

Vamos supor agora que $X \subset \omega(=A), X \notin \mathcal{A}$ e $\mathcal{A} \cup\{X\}$ é uma família independente. Então para todo $p \in F n(\mathcal{A}, 2) \backslash\{\emptyset\}$ temos que:

$W(p) \cap X \neq \emptyset \neq W(p) \cap(A \backslash X)$.

Assim mostramos que o espaço $A$ será resolúvel se e somente se a família independente $\mathcal{A}$ não for maximal.

\section{Teorema 3.1.5 $i=\lambda$}

Prova. Primeiro vamos mostrar que $i \leq \lambda$.Escolhemos um subespaço denso, enumerável e irresolúvel $A \subset\{0,1\}^{\lambda}$ no cubo de Cantor de peso $\lambda$. Do lema 3.1.4, temos a família independente maximal $\mathcal{A}=\{W(\alpha, 0) \cap A: \alpha<\lambda\}$ de cardinalidade $|A|=\lambda$, portanto $i \leq \lambda$.

Agora vamos provar que $\lambda \leq i$. Seja $\mathcal{A}=\{V(\alpha): \alpha \in$ i\} $\subset p(\omega)$ uma família independente maximal de cardinalidade $i$. Vamos supor que $\alpha \neq \beta \Longrightarrow V(\alpha) \neq V(\beta)$ para todo $\alpha, \beta \in i$.

Do lema 3.1.3, seja $A=\psi_{\mathcal{A}}[\omega]$ um subespaço denso e enumerável do cubo de Cantor $2^{\mathcal{A}}$. Precisamos mostrar que $A$ é um espaço irresolúvel. Por absurdo, suponha que $D_{0}, D_{1}$ são dois subconjuntos densos e disjuntos do espaço $A$, e que satisfazem $D_{0} \cup D_{1}=A$. Então $\forall p \in F n(i, 2), W(p) \cap D_{0} \neq \emptyset \neq W(p) \cap D_{1}$ e assim temos que $\mathcal{A} \subset \mathcal{A} \cup\left\{\psi_{\mathcal{A}}{ }^{-1}\left(D_{0}\right)\right\}=\mathcal{B}$ e para todo $p \in F n(i, 2)$ e $V(p)=(\bigcap\{V(\alpha) \in \mathcal{A}: p(\alpha)=0\}) \cap(\bigcap\{\omega \backslash V(\alpha):$ $p(\alpha)=1\})$ onde $\alpha \in \operatorname{dom}(p)$ temos $V(p) \cap \psi_{\mathcal{A}}{ }^{-1}\left(D_{0}\right) \neq \emptyset \neq$ 
$V(p) \cap\left(\omega \backslash \psi_{\mathcal{A}}{ }^{-1}\left(D_{0}\right)\right)$. Assim mostramos que $\mathcal{B}$ é uma família independente de $p(\omega)$. Contradição, uma vez que $\mathcal{A}$ é maximal. Logo $A$ é um subespaço denso, enumerável e irresolúvel do cubo $\{0,1\}^{i}$. Assim $\lambda \leq i$.

A proposição que vem a seguir, é um fato que complementa as nossas observações a respeito das imersões densas nos cubos de Cantor. Ela está diretamente ligada à proposição 2.2.6.

Proposição 3.1.6 Fixamos um espaço regular e enumerável $X=$ $(\omega, \tau)$ com peso $w(X)=\aleph_{1}$ e pi-carácter local $\pi \chi(x, X)=\aleph_{1}$ para todo $x \in X$. Para cada sub-álgebra enumerável $\mathcal{A} \subset \mathcal{D}_{0}(X)$, que separa pontos de $\omega$, denotamos por $Y_{\mathcal{A}}=\left(\omega, \tau_{\mathcal{A}}\right)$, o correspondente espaço regular. Se $\pi: \beta X \longrightarrow \beta Y_{\mathcal{A}}$ é aberta,então $X$ é densamente imersível em $2^{\aleph_{1}}$.

Prova. Enumeramos a família de todos os pares $(x, F)$, formados por um ponto $x$ e um fechado $F$ de $X$, ao qual o ponto não pertence, na forma $\left\{P_{t}: t \in c\right\}$, onde $P_{t}=\left(x_{t}, F_{t}\right)$.Seja $\mathcal{B}=$ $\left\{W_{\beta}: \beta \in \aleph_{1}\right\}$ uma base de abertos-fechados do espaço $X$.

Por indução transfinita, vamos construir uma sequência transfinita de sub-álgebras enumeráveis $\left\{\mathcal{A}_{\alpha}: \alpha<\aleph_{1}\right\}$,tal que:

(1) $\mathcal{A}_{0} \subset \mathcal{D}_{0}(X)$, separa pontos de $X$.

(2) $\mathcal{A}_{\alpha+1}=\left[\mathcal{A}_{\alpha} \cup\left\{T_{\alpha+1}\right\}\right]$, com int $\operatorname{in}_{\mathcal{A}_{\alpha}}\left(T_{\alpha+1}\right)=\emptyset$.

(3) Se $\Gamma$ é ordinal limite, então $\mathcal{A}_{\Gamma}=\cup\left\{\mathcal{A}_{\alpha}: \alpha \in \Gamma\right\}$.

(4) $\mathcal{A}=\cup\left\{\mathcal{A}_{\alpha}: \alpha \in \aleph_{1}\right\}$, é base de abertos de $X$.

Suponhamos que $\mathcal{A}_{\alpha}$ está escolhida. Seja $t(\alpha)=\min \{t$ : $\mathcal{A}_{\alpha}$ não separa $\left.P_{t}\right\}$, e seja $\beta(\alpha)=\min \left\{\beta: W_{\beta}\right.$ separa $\left.P_{t(\alpha)}\right\}$. Usando a proposição 2.2.6, seja $\mathcal{A}_{\alpha+1}=\left[\mathcal{A}_{\alpha} \cup\{T, X \backslash T\}\right]$,onde $T \in \mathcal{D}_{0}(X)$ é tranversal à $\mathcal{A}_{\alpha}$ e existe $V \in \mathcal{D}_{0}\left(Y_{\mathcal{A}_{\alpha}}\right)$, tal que $V \cap T=W_{\beta(\alpha)} \backslash i n t_{Y_{\alpha}}\left(W_{\beta(\alpha)}\right)$, usando a nota 2.2.7. No caso em 
que $\Gamma \in \aleph_{1}$ é limite, tomamos $\cup\left\{\mathcal{A}_{\alpha}: \alpha \in \Gamma\right\}$, completando a indução. Seja $\mathcal{A}=\bigcup_{\alpha \in \aleph_{1}} \mathcal{A}_{\alpha}$ a álgebra resultante.

Como fizemos a escolha, em cada passo da indução, $\mathcal{A}_{\alpha+1}=$ $\left[\mathcal{A}_{\alpha} \cup\{T, X \backslash T\}\right]$, com este transversal $T$ tal que $V \cap T=$ $W_{\beta(\alpha)} \backslash i n t_{Y_{\alpha}}\left(W_{\beta(\alpha)}\right)$, a álgebra resultante, separa todos os pares $P_{t}$, que a subfamília $\left\{W_{\beta}: \beta \leq \beta(\alpha)\right\}$ já separavam. Logo $\mathcal{A}$ separa todos os pares $P_{t}$ formados por um ponto $x$ e um fechado $F$ ao qual este ponto não pertence, isto é; $\mathcal{A}$ é base do espaço $X$.

Se $\mathcal{T}_{\alpha}$ é uma "f.i." que gera $\mathcal{A}_{\alpha}$ então $\mathcal{T}_{\alpha} \cup\{T\}=\mathcal{T}_{\alpha+1}$ é uma "f.i." que gera $\mathcal{A}_{\alpha+1}$. Assim, a imersão de $X$ por meio da "f.i" $\mathcal{T}=\cup\left\{\mathcal{T}_{\alpha}: \alpha \in \aleph_{1}\right\}$, usando o lema 3.1.3, é uma cópia densa em $2^{\aleph_{1}}$ do espaço $X$.

O teorema 3.1.5 é a caracterização topológica das famílias independentes maximais. Como consequência desta caracterização, e das proposições 2.1.3 e 2.1.4, e do fato que todo abertofechado básico $\emptyset \neq W \subset 2^{\kappa}$ é uma cópia de $2^{\kappa}$, podemos afirmar que existem "f.i." maximais $\mathcal{A}$ tais que, se $V \in \mathcal{A}$ então $\{T \cap V: T \in \mathcal{A}\}$, ainda é uma "f.i."maximal. Tal fato equivale à proposição 2.1.3.

Vamos a seguir dar uma caracterização dos espaços regulares enumeráveis densos em si mesmos, densos no cubo de Cantor de peso $c$, e que são espaços submaximais, nos termos das "f.i."maximais. É uma forte aplicação do teorema 3.1.5.

\subsection{Espaços Submaximais}

Em [ASTTW] os autores mostram que no cubo de Tychonof $[0,1]^{c}$, dentro de um modelo de ZFC mais BL ( Booth Lemma 
ou $p=c$ ), existem subespaços densos enumeráveis submaximais.Também é bem conhecido [Vau] que $p \leq i$ e que $p<i$ é consistente. Malykhin [Ma]mostrou que num modelo de BellKunen o cubo de Cantor de peso $\omega_{1}$ contém um subespaço denso, enumerável e irresolúvel. Assim pelo teorema 3.1.5 é verdade que $i=\omega_{1}$, dentro do modelo de Bell-Kunen. Mostraremos que neste modelo, onde o continuum $c=\aleph_{\omega_{1}}$, o cubo de Cantor de peso $c$ contém um subespaço denso enumerável submaximal. Assim a existência de tais subespaços densos enumeráveis e sumaximais no cubo $2^{c}$ é independente de $i=c$ ou $p=c$.

Seja $\mathcal{A}$ uma família independente de $p(\omega)$. Nesta secção vamos supor sempre que $X=(\omega, \tau)$ é um espaço topológico cuja subbase é a família $\mathcal{A} \cup\{\omega \backslash V: V \in \mathcal{A}\}$, e $A=\psi_{\mathcal{A}}[\omega]$ é um subespaço denso do cubo de Cantor $\{0,1\}^{\mathcal{A}}$. Também fixamos a notação $\mathcal{A}=\left\{V_{\alpha}: \alpha \in \kappa\right\}$, onde supomos que $|\mathcal{A}|=\kappa$.

Recordamos que um espaço topológico $(X, \tau)$ é um espaço submaximal se todo subconjunto denso $D \subset X$ é um subconjunto aberto de $X$.

Para cada $p \in F n(\kappa, 2)$ denotamos por $V(p)$ ao subconjunto $\left(\cap\left\{V_{\alpha}: p(\alpha)=0\right\}\right) \bigcap\left(\cap\left\{\omega \backslash V_{\alpha}: p(\alpha)=1\right\}\right)$. Em particular se $p=\emptyset$, então vamos supor que $V(p)=\omega$.

Definição 3.2.1 Dizemos que um subconjunto $D \subset \omega$ é denso em $\mathcal{A}$ se $|D \cap V(p)|=\omega$ para todo $p \in F n(\kappa, 2)$. Dizemos que um subconjunto $G \subset \omega$ é um aberto em $\mathcal{A}$ se $\forall x \in G, \exists p \in F n(\kappa, 2)$ tal que $x \in V(p) \subset G$.

Proposição 3.2.2 As seguintes afirmações são equivalentes:

(i) $D$ é denso em $\mathcal{A}$, 
(ii) $\mathcal{A}(D)=\left\{V_{\alpha} \cap D: \alpha \in \kappa\right\}$ é uma família independente de $p(D)$ (partes de $D)$,

(iii) $\psi_{\mathcal{A}}[D]$ é denso em $A$.

Com efeito, suponhamos que $D \subset \omega$ é denso em $\mathcal{A}$. Para $p \in F n(\kappa, 2)$ temos $\left(\cap\left\{D \cap V_{\alpha}: p(\alpha)=0\right\}\right) \bigcap\left(\cap\left\{D \backslash V_{\alpha}: p(\alpha)=\right.\right.$ $1\})=D \cap V(p)$, e este último é infinito. Mas, se $\mathcal{A}(D)$ é uma "f.i" então da igualdade acima segue a afirmação (i). Que (i) é equivalente a (iii) são os lemas 3.1 .3 e 3.1.4 e a definição de $\psi_{\mathcal{A}}$.

Proposição 3.2.3 São equivalentes as seguintes afirmações:

(1) $G \subset \omega$ é aberto em $\mathcal{A}$

(2) $G$ é aberto em $X$

(3) $\psi_{\mathcal{A}}[G]$ é aberto em $A$.

Também, esta proposição é imediata.

Vamos descrever a seguir uma família independente enumerável, que será útil nas construções futuras de outras famílias independentes, que faremos, para mostrar que existem subespaços densos enumeráveis e submaximais no cubo de Cantor de peso $c$, sob certas condições especiais,isto é, em algum modelo de ZFC.

Exemplo 3.2.4 Fixemos o subespaço denso e enumerável $Z=$ $\{z:\{n: z(n) \neq 0\}$ é finito $\} \subset 2^{\omega}$. Escolhemos $s \in 2^{\omega}$ de modo que $|\{n: s(n)=0\}|=|\{n: s(n)=1\}|$. Seja $\mathcal{T}$ a familia independente definida por $\mathcal{T}=\left\{T_{\alpha}: \alpha \in \omega\right\}$, onde $\forall \alpha \in \omega, T_{\alpha}=$ $Z \cap W(\alpha, s(\alpha))$, e como sempre, $W(\alpha, i)=\left\{s \in 2^{\omega}: s(\alpha)=i\right\}$ para todo $\alpha \in \omega$, e $i \in 2$. Então $\mathcal{T}$ satisfaz: $\cap \mathcal{T}=\emptyset$ bem como $\bigcup \mathcal{T}=Z$.

O lema que segue é imediato. 
Lema 3.2.5 Sejam $\mathcal{A}$ e $\mathcal{B}$ duas famílias independentes, com $\mathcal{A} \subset \mathcal{B}$. Então,

(i) $D \subset \omega$ é denso em $\mathcal{B}$ implica $D$ também é denso em $\mathcal{A}$;

(ii) $G \subset \omega$ é aberto em $\mathcal{A}$ implica $G$ também é aberto em $\mathcal{B}$.

Lema 3.2.6 Seja D um subconjunto de $\omega$ que satisfaz as seguintes condições:

(i) $D=\bigcup_{n \in \omega} D_{n}$ para todo $n \in \omega,\left|D_{n}\right| \geq 1$,

(ii) $n \neq m \Longrightarrow D_{n} \cap D_{m}=\emptyset$.

Seja $\mathcal{T}$ a familia independente do exemplo 3.2.4. Então

$\mathcal{D}=\left\{V_{\alpha}: \alpha<\omega\right\}$ dada por $V_{\alpha}=\bigcup\left\{D_{n}: n \in T_{\alpha}\right\}$ é uma familia independente de $p(\omega)$ e $D$ é aberto em $\mathcal{D}$.

Prova. Dado $p \in F n(\omega, 2)$, temos que $x \in V(p)(\operatorname{pg} 35)$ se e somente se $\forall \alpha \in \operatorname{dom}(p), \exists n \in T(\alpha, p(\alpha))$, tal que $x \in D_{n}$, por (i) e (ii), se e somente se, $\exists n \in T(p)$, tal que $x \in D_{n}$. Onde, $T(\alpha, p(\alpha))=T_{\alpha}$, se $p(\alpha)=0$, ou $T(\alpha, p(\alpha))=\omega \backslash T_{\alpha}$, se $p(\alpha)=1$. Assim, $V(p)=\bigcup\left\{D_{n}: n \in T(p)\right\}$, onde $T(p)=$ $\bigcap_{\alpha \in \operatorname{dom}(p)} T(\alpha, p(\alpha))$.

Relembramos que um espaço $X$ é $\omega$-resolúvel se existe uma família infinita ( enumerável ) de subconjuntos densos em $X$, dois a dois disjuntos.

Lema 3.2.7 Seja $\mathcal{A}$ uma familia independente e suponhamos $q u e|\mathcal{A}|<i$. Se $D \subset \omega$ é denso em $\mathcal{A}$ então existe uma sequência $\left\{D_{n}: n \in \omega\right\}$ de subconjunto de $D$ tal que:

(i) para cada $n \in \omega, D_{n}$ é denso em $\mathcal{A}$,

(ii) $n \neq m \Longrightarrow D_{n} \cap D_{m}=\emptyset$,

(iii) $\bigcup_{n \in \omega} D_{n}=D$

Prova. Como $|\mathcal{A}|<i$, o subespaço denso $\psi_{\mathcal{A}}[D] \subset 2^{\mathcal{A}}$ é resolúvel, e se $G \subset \psi_{\mathcal{A}}[D]$ é denso em $\psi_{\mathcal{A}}[D]$, então este $G$ também é 
resolúvel. Assim $\psi_{\mathcal{A}}[\omega]$ é $\omega$-resolúvel, e portanto $D$ é $\omega$-resolúvel. ( Usamos a proposição 3.2.2 ).

Lema 3.2.8 Seja $D \subset \omega$ denso em $\mathcal{A}$ e suponhamos que $|\mathcal{A}|<$ i. Então existe uma família independente $\mathcal{B} \subset p(\omega)$ tal que:

(i) $\mathcal{A} \subset \mathcal{B}$ e $|\mathcal{B} \backslash \mathcal{A}|=\omega$,

(ii) $D$ é denso e aberto em $\mathcal{B}$.

Prova. Escolhemos $\left\{D_{n}: n \in \omega\right\}$ satisfazendo as condições do lema 3.2.7, e então seja, $\mathcal{D}$ a família independente definida no lema 3.2.6. Definimos $\mathcal{B}=\mathcal{A} \cup \mathcal{D}$, que é uma família independente, e como, $D=\cup \mathcal{D}$, é claro que $D$ é aberto em $\mathcal{B}$. Vamos introduzir uma notação.

Para cada $p \in F n(\mathcal{B}, 2)$, podemos fixar $s \in F n(\mathcal{A}, 2)$ e $t \in F n(\mathcal{D}, 2)$ tais que

$s=p\lceil\mathcal{A} \cap \operatorname{dom}(p)$ e $t=p\lceil\mathcal{D} \cap \operatorname{dom}(p)$

e assim, se $W \in[\mathcal{B}]$, isto é; $W$ é um elemento da álgebra finitamente gerada por $\mathcal{B}$; podemos supor que $W=B(p)=$ $\left(\cap\left\{B_{\alpha}: p(\alpha)=0\right\}\right) \bigcap\left(\cap\left\{\omega \backslash B_{\alpha}: p(\alpha)=1\right\}\right)$ onde, de modo análogo, $A(s) \cap D(t)=W$, são definidos por $A(s)=\left(\cap\left\{A_{\alpha}\right.\right.$ : $s(\alpha)=0\}) \bigcap\left(\cap\left\{\omega \backslash A_{\alpha}: s(\alpha)=1\right\}\right)$, e $D(t)=\left(\cap\left\{D_{\alpha}: t(\alpha)=\right.\right.$ $0\}) \cap\left(\cap\left\{\omega \backslash D_{\alpha}: t(\alpha)=1\right\}\right)$.

Como para cada $n \in \omega$ cada $D_{n}$ é denso em $\mathcal{A}$, temos que $\left|A(s) \cap D_{n}\right|=\omega$, e assim, $|W \cap D|=\omega$.

O nosso principal corolário do teorema de caracterização do cardinal $i$ é a tradução da existência de subespaços densos enumeráveis submaximais no cubo de Cantor $2^{c}$, nos termos das álgebras de Boole, que vem logo a seguir.

Teorema 3.2.9 $(i=c)$ Existe uma família independente $\mathcal{A} \subset$ $p(\omega)$ tal que: 
(i) $|\mathcal{A}|=c e$

(ii) se $D \subset \omega$ é denso em $\mathcal{A}$ então $D$ é aberto em $\mathcal{A}$.

Prova.Seja $\left\{E_{\alpha}: \alpha<c\right\}$ uma enumeração de todos os subconjuntos infinitos de $\omega$ com $E_{0}=\omega$ e escolhamos uma família independente $\mathcal{A}_{0}$ com cardinalidade $\left|\mathcal{A}_{0}\right|<c$ e $\cup \mathcal{A}_{0}=\omega$. Por indução transfinita em $c \backslash\{0\}$ vamos definir uma sequência $\left\{\mathcal{A}_{\alpha}: \alpha \in c \backslash\{0\}\right\}$ de famílias independentes tais que:

(i) $\mathcal{A}_{\alpha} \subset \mathcal{A}_{\alpha+1}$

(ii) $\left|\mathcal{A}_{\alpha+1} \backslash A_{\alpha}\right|=\omega$

(iii)se $E_{\alpha}$ é denso em $\mathcal{A}_{\alpha}$ então $E_{\alpha}$ é denso e aberto em $\mathcal{A}_{\alpha+1}$ usando o lema 3.2.8 para definir estes conjuntos, e

(iv) se $\beta$ é limite então seja $\mathcal{A}_{\beta}=\bigcup_{\alpha \in \beta} \mathcal{A}_{\alpha}$.

A família independente que fica definida por $\mathcal{A}=\bigcup_{\alpha \in c} \mathcal{A}_{\alpha}$

tem cardinalidade $|\mathcal{A}|=c$ e se $D \subset \omega$ é denso em $\mathcal{A}$ então $\exists \alpha \in c$ tal que $D=E_{\alpha}$ e este conjunto é denso em $\mathcal{A}_{\alpha}$ portanto é também aberto em $\mathcal{A}_{\alpha+1}$, pela construção por indução, assim é ainda aberto em $\mathcal{A}$.

Corolário 3.2.10 $(i=c) O$ cubo de Cantor $\{0,1\}^{c}$ contem um subespaço denso enumerável submaximal.

Prova.Escolhemos uma família independente $\mathcal{A}$ que satisfaz o teorema 3.2.9. Seja $\psi_{\mathcal{A}}[\omega]=A$ o subespaço denso do cubo de Cantor de peso $c$. Se $D$ é denso em $A$ então $\psi_{\mathcal{A}}{ }^{-1}(D)$ é denso em $\mathcal{A}$, assim também é denso e aberto em $\mathcal{A}$ e portanto $D$ é aberto em $A$. 


\subsection{Modelo de Bell e Kunen}

Seja $M$ um modelo transitivo enumerável para ZFC mais GCH. Bell e Kunen [BK] construiram em $M$ uma família crescente de ordens parciais $\left\{P_{\alpha}: \alpha \leq \omega_{1}\right\}$ com as seguintes propriedades:

(i) cada $P_{\alpha}$ tem c.c.c.

(ii) se $\beta$ é limite $P_{\beta}=\cup\left\{P_{\alpha}: \alpha<\beta\right\}$

(iii) se $\alpha$ não é limite então $P_{\alpha}$ é tal que MA (axioma de Martin ) é verificado em $M^{P_{\alpha}}$.

Seja $G=G_{\omega_{1}}$ um filtro $P_{\omega_{1}}$-genérico sobre $M$ e $G_{\alpha}=G \cap P_{\alpha}$ para cada $\alpha \leq \omega_{1}$. Em $M\left[G_{\omega_{1}}\right]$ existe uma sequência crescente transfinita de modelos $\left\{M\left[G_{\alpha}\right]: \alpha \leq \omega_{1}\right\}$, e se $\alpha>0$ é um ordinal não limite então em $M\left[G_{\alpha}\right]$ valem MA e $c=\aleph_{\alpha}$. Observamos também, que em $M\left[G_{\omega_{1}}\right]$, o conjunto das partes de $\omega$ é a reunião da sequência crescente $\left\{p(\omega) \cap M\left[G_{\alpha+1}\right]: \alpha<\omega_{1}\right\}$.

Teorema 3.3.1 No modelo de Bell e Kunen, existe uma família independente $\mathcal{A} \subset p(\omega)$ tal que todo subconjunto $D \subset \omega$ denso em $\mathcal{A}$ é aberto em $\mathcal{A}$.

Prova. Por indução transfinita dentro de $M\left[G_{\omega_{1}}\right]$, vamos construir uma sequência crescente de famílias independentes $\left\{\mathcal{A}_{\alpha}\right.$ : $\left.\alpha<\omega_{1}\right\}$, tais que dentro de $M\left[G_{\alpha+1}\right]$, a família $\mathcal{A}_{\alpha}$ satisfaz o teorem 3.2.9. Isto é possivel porque dentro de $M\left[G_{\alpha+1}\right]$ vale MA , então $i=c=\aleph_{\alpha}$. Agora olhamos esta família dentro de $M\left[G_{\alpha+2}\right]$, e dentro deste modelo temos que $\left|\mathcal{A}_{\alpha+1}\right| \leq$ $\aleph_{\alpha+1}<\aleph_{\alpha+2}=c$, e então pela prova do teorema 3.2.9, dentro de $M\left[G_{\alpha+2}\right]$, podemos escolher uma família independente $\mathcal{A}_{\alpha+2} \supset \mathcal{A}_{\alpha+1}$, e tal que dentro de $M\left[G_{\alpha+2}\right]$ se $D$ é denso em $\mathcal{A}_{\alpha+2}$ então também é aberto. (Para um ordinal limite tomamos a reunião ). A família $\mathcal{A}=\cup\left\{\mathcal{A}_{\alpha}: \alpha<\omega_{1}\right\}$ é uma família independente dentro de $M\left[G_{\omega_{1}}\right]$ e se $D \subset \omega$ é denso em $\mathcal{A}$ então 
$\exists \alpha<\omega_{1}$ tal que $D \in p(\omega) \cap M\left[G_{\alpha+1}\right]$ e assim $D$ é aberto em $A_{\alpha+2}$ dentro $M\left[G_{\alpha+2}\right]$, portanto é aberto em $\mathcal{A}$ dentro de $M\left[G_{\omega_{1}}\right]$.

Corolário 3.3.2 No modelo de Bell e Kunen há um subespaço denso enumerável submaximal do cubo de Cantor $\{0,1\}^{c}$.

Prova. Tomamos uma família independente $\mathcal{A}$ como no teorema 3.3.1. Seja $\psi_{\mathcal{A}}[\omega]$ um subespaço denso enumerável submaximal de $2^{c}$.

Malyhin mostrou que neste modelo valem os fatos:

Fato 3.3.3 (Ma) No modelo de Bell e Kunen existe um grupo booleano enumerável com topologia maximal. Também neste modelo, o cubo de Cantor $2^{\omega_{1}}$ contem um subespaço denso enumerável e irresolúvel.

Fato 3.3.4 (BK) Seja $\beta \omega \backslash \omega=\omega^{*}$. No modelo de Bell e Kunen, $\forall p \in \omega^{*}$ vale $\pi \chi\left(p, \omega^{*}\right)=\aleph_{1}$.

3.3.5 A existência de um subespaço denso enumerável submaximal $X \subset 2^{c}$, foi estabelecida, em modelos onde valem: $B L$, $M A, i=c$, e no modelo de Bell e Kunen, onde $i=\aleph_{1}<c=\aleph_{\omega_{1}}$.

\subsection{Grupos Booleanos enumeráveis}

O objetivo desta secção, é mostrar que se dois subgrupos densos e enumeráveis $G, H$ do cubo de Cantor $2^{c}$ tem a topologia de Bohr como subespaço de $2^{c}$ então existe um isomorfismo bicontínuo $\psi$ do cubo de Cantor $2^{c}$ tal que $\psi[G]=H$. Vamos estabelecer uma condição suficiente para imersões densas nos cubos de Cantor de peso $\kappa, \aleph_{1} \leq \kappa \leq c$. 
Um grupo booleano $(G,+)$ é um grupo abeliano cujos elementos tem ordem 2. Podemos considerar estes grupos $G$ como espaços vetoriais sobre o corpo $Z_{2}=2$. Assim é fácil ver que $G, H$ são isomorfos se e somente se $|G|=|H|$.

Seja $G$ um grupo booleano com $|G|=\kappa$. Um conjunto de vetores linearmente independente $B \subset G$ é uma base de $G$ se e somente $<B>=G$. Sejam $A=\left\{a_{\alpha}: \alpha<\kappa\right\}, B=\left\{b_{\alpha}: \alpha<\kappa\right\}$ bases de dois grupos booleanos $G, H$ de mesma cardinalidade. A função $\varphi: G \longrightarrow H$ definida por $\varphi\left(a_{\alpha}\right)=b_{\alpha}$ estendida linearmente é um isomorfismo linear entre $G$ e $H$.

Definição 3.4.1 Seja $(G,+, \tau)$ um grupo booleano topológico enumerável denso em si mesmo. Denotamos por hom $(G)$ o conjunto de todos os homomorfismos de $G$ em $\{0,1\}$. Dizemos que $G$ é um grupo pseudo-maximal se todos os homomorfismos $f \in \operatorname{hom}(G)$ forem funções contínuas.

O conjunto $\operatorname{hom}(G)$ é ele mesmo um grupo booleano se definirmos para todo par de homomorfismo $f, g \in h o m(G)$ e para cada $x \in G$ a soma por $(f+g)(x)=f(x)+g(x)$.

Vamos denotar por $K H O M(G)$ o conjunto $\left\{f^{-1}(0): f \in\right.$ hom $(G)\}$ que é o conjunto de todos os subgrupos $W \subset G$ tais que se $x, y \in G \backslash W$ então $x+y \in W$. Se $G$ é um grupo booleano finito então $|G|=|K H O M(G)|=2^{n}$ para algum $n \in \omega$. (Para cada $W \in K \operatorname{HOM}(G)$ existe um e somente um homormofismo $h \in h o m(G)$ tal que $\left.h^{-1}(0)=W\right)$. Observamos que hom $(G)$ é o dual algébrico de $G$, assim $|G|=2^{n}$ se e somente se $|h o m(G)|=$ $2^{n}$.

Proposição 3.4.2 Seja $G$ um grupo booleano infinito enumerável e seja $\mathcal{D}$ um conjunto não vazio contido em hom $(G)$. São equivalentes as seguintes afirmações: 
(i) $\mathcal{D}$ é um conjunto linearmente independente em hom $(G)$;

(ii) $\left\{f^{-1}(0): f \in \mathcal{D}\right\}$ é uma família independente de subgrupos de $G$.

Prova. (i) $\Longrightarrow$ (ii). Seja $F=\left\{f_{1}, \ldots, f_{n}\right\}$ um subconjunto finito de $\mathcal{D}$. Precisamos mostrar que para todo $p \in 2^{F}$ cada um dos subconjuntos $W(p)=\bigcap_{f \in F} f^{-1}(p(f))$, é um subconjunto infinito de $G$.

Primeiro vamos observar que $F$ é um conjunto linearmente independente se e somente se $|\langle F\rangle|=2^{n}$. Com efeito, para $\{1, \ldots, n\}=I$ o subgrupo de $h o m(G)$ que é gerado por $F$ é dado por: $\langle F\rangle=\left\{h_{s}: s \in 2^{I}\right\}$ onde $h_{s}=\sum_{i \in I} s(i) f_{i}=\sum_{s(i)=1} f_{i}$. Então $|\langle F\rangle|=2^{n}$ se e somente se $h_{s}=0 \Longrightarrow s=0$ se e somente se $F$ é um conjunto linearmente independente.

Agora, seja $W=\bigcap_{f \in F} f^{-1}(0) \subset G$ o subgrupo fixado por $F$. Definimos a função $\pi: G \longrightarrow 2^{F}$ tal que $\pi(x)(f)=f(x)$ para todo $x \in G$ e todo $f \in F$. Tomamos, $A=\pi[G] \subset 2^{F}$ a imagem do homomorfismo $\pi$, e então $A$ é um subgrupo de $2^{F}$, tal que:

$\forall p \in A, W(p)=\bigcap_{f \in F} f^{-1}(p(f))=\pi^{-1}(p)=x+W$ para todo $x \in G$ tal que $\pi(x)=p$. Se $y \in x+W$ então $x+y \in$ $W \Longrightarrow \pi(x+y)=0$ ou $\pi(x)=\pi(y)$.

O subgrupo $A$ é isomorfo ao grupo quociente

$\frac{G}{W}=\{x+W: x \in G\}=\{W(p): p \in A\}$.

Precisamos mostrar que $A=2^{F}$. Seja $h=h_{s}$ um ponto de $<F>$. Então temos:

$W \subset \bigcap_{s(i)=1} f_{i}^{-1}(0) \subset h^{-1}(0)$, então, definimos a função, $\psi$ : $2^{I} \longrightarrow K H O M(A)$ tal que $\psi(s)=A_{s}=\{p \in A: W(p) \subset$ $\left.h^{-1}(0)\right\}$, onde pelo teorema do isomorfismo da álgebra linear, te$\operatorname{mos}, \frac{h^{-1}(0)}{W}=\left\{x+W: x \in h^{-1}(0)\right\}=\left\{W(p): p \in \pi\left[h^{-1}(0)\right]\right\} \subset$ $\frac{G}{W}$, portanto $A_{s}$ é o subgrupo $\pi\left[h^{-1}(0)\right]=A_{s} \subset A$, e ele é tal que $p, q \in A \backslash A_{s}$ implica $p+q \in A_{s}$. Com efeito, escolhemos 
$x, y \in G \backslash h^{-1}(0)$ tal que $\pi(x)=p$ e $\pi(y)=q$ então $p+q=$ $\pi(x+y)$, mas $x+y \in h^{-1}(0)$. Portanto, $A_{s} \in K \operatorname{HOM}(A)$. Se $s, t \in 2^{I}$ e $A_{s}=A_{t}$ então $\pi^{-1}\left(A_{s}\right)=h_{s}{ }^{-1}(0)=h_{t}{ }^{-1}(0)=$ $\pi^{-1}\left(A_{t}\right) \Longrightarrow h_{s}=h_{t} \Longrightarrow s=t$. Assim mostramos que $\psi$ é uma função injetora, logo

$$
2^{n}=|<F>| \leq|h o m(A)|=|\operatorname{KHOM}(A)| \leq 2^{n}
$$

portanto, $A=2^{F}$.

(ii) $\Longrightarrow$ (i). Para um $p \in F n(\mathcal{D}, 2) \backslash\{\emptyset\}$ vale que $W(p)$ é um subconjunto infinito de $G$. Seja $h=\sum_{f \in \operatorname{dom}(p)} f$. Então $h^{-1}(1)=\bigcup\left\{W(q): \operatorname{dom}(q)=\operatorname{dom}(p), \sum_{f \in \operatorname{dom}(p)} q(f)=1\right\} \neq \emptyset$.

Corolário 3.4.3 Seja $\mathcal{D} \subset$ hom $(G)$ um conjunto linearmente independente São equivalentes as seguintes condições:

(1) $\mathcal{D}$ é maximal como conjunto linearmente independente em hom $(G)$

(2) $\langle\mathcal{D}\rangle=\operatorname{hom}(G)$

(3) $\{\operatorname{ker}(f): f \in h o m(G)\}$ é uma família independente maximal de subgrupos de $G$.

Assim, se $\mathcal{D}$ é maximal então $|\mathcal{D}|=c$.

Agora, vamos fixar um grupo topológico booleano infinito enumerável $(G,+, \tau)$. Seja $\mathcal{D}$ uma base para hom $(G)$ e definimos a função:

$\psi: G \longrightarrow 2^{\mathcal{D}}$ tal que $\psi(x)(f)=f(x) ; \forall x \in G, f \in \mathcal{D}$. Tomamos a sua imagem, e por (3) do corolário acima, $\psi[G]=H \subset 2^{\mathcal{D}}$ é um subgrupo topológico denso do cubo de Cantor $\{0,1\}^{\mathcal{D}}$ de peso $|\mathcal{D}|=c$. 
Proposição 3.4.4 A função $\psi$ definida acima, é um isomorfismo de $G$ sobre $H$. Se a topologia de $G$ é pseudo-maximal então $\psi$ é uma função contínua, onde $H$ tem a topologia de subespaço de $2^{\mathcal{D}}$.

Proposição 3.4.5 Se G tem a topologia de Bohr e H tem a topologia de subespaço de $2^{\mathcal{D}}$, então $\psi: G \longrightarrow H$ é um homeomorfismo.

Corolário 3.4.6 Existe um subgrupo topológico denso enumerável pseudo-maximal $H \subset 2^{c}$.

Proposição 3.4.7 Se G tem uma topologia irresolúvel então $G$ é um grupo topológico pseudo-maximal.

Prova. Suponhamos, por absurdo, que $h \in h o m(G)$ é um homomorfismo tal que $h^{-1}(0)=W$ é um subgrupo com $i n t_{G}(W)=\emptyset$. Definimos o homeomorfismo $\psi_{a}: G \longrightarrow G$ tal que $\psi_{a}(x)=x+a$ para todo $x \in G$, onde $a \in G \backslash W$. Então, temos $\psi_{a}[W]=$ $G \backslash W$, e portanto ambos os subconjuntos $W$ e $G \backslash W$ são densos em $G$. (Contradição, pois $G$ é um espaço irresolúvel). Assim $\operatorname{int}_{G}(W) \neq \emptyset$ e isto mostra que $W$ e $G \backslash W$ são subconjuntos abertos e fechados de $G$. Logo $h$ é uma função contínua.

O fato que usamos $\operatorname{int}_{G}(W) \neq \emptyset$ implica que $W$ é um abertofechado, é devido a $W$ ser um subgrupo do grupo topológico $G$. [ Kelley, pg 106,T,c].

Nota 3.4.8 (Fréchet e Brouwer, 1910)

Sejam $A, B$ dois subespaços densos e enumeráveis da reta real $\mathbb{R}$. Existe um homeomorfismo $h: \mathbb{R} \rightarrow \mathbb{R}$ tal que $h[A]=B$.

Há inúmeros problemas em aberto, até os dias de hoje, que pedem alguma generalização deste fato, [Op]. Os espaços onde valem fatos análogos, que são conhecidos, são o conjunto de 
Cantor $2^{\omega}$, e o espaço dos números irracionais $P \subset \mathbb{R}$, e o nosso teorema acima, nos dá mais um exemplo, o único que conhecemos, na classe dos espaços não metrizáveis.

Alguma técnica, deve ser desenvolvida, para uma abordagem nesta classe de problemas, e assim, devemos estudar as possiveis topologias, que podemos definir, nos espaços dos homeomorfismos, de um espaço sobre si mesmo, com boas propriedades, tais como serem homogêneos, etc.

Proposição 3.4.9 Sejam $A, B$ dois subespaços densos do cubo de Cantor $2^{c}$.São equivalentes as seguintes afirmações:

(i) existe um homeomorfismo $\psi: 2^{c} \longrightarrow 2^{c}$ tal que $\psi[A]=B$,

(ii) existe um homeomorfismo $\varphi: A \longrightarrow B$ tal que $\mathcal{D}\lceil B=$ $\{\varphi[T]: T \in \mathcal{D}\lceil A\}$ onde $\mathcal{D}$ denota a álgebra booleana de todos os subconjuntos abertos e fechados de $2^{c}$ e $\mathcal{D}\lceil X=\{W \cap X: W \in$ $\mathcal{D}\}$.

Lema 3.4.10 Seja $G \subset 2^{c}$ um subgrupo denso, enumerável e pseudo-maximal do cubo de Cantor de peso c. Para cada $\alpha \in c$ definimos $h_{\alpha}: G \longrightarrow 2$ tomando $\forall x \in G, h_{\alpha}(x)=x(\alpha)$. Então $<h_{\alpha}: \alpha \in c>=\operatorname{hom}(G, 2)$.

Prova. Como $G$ é subespaço denso, a família definida por $\left\{h_{\alpha}{ }^{-1}(0): \alpha \in c\right\}$ é uma família independente; assim pela proposição 3.4.2, o conjunto $B=\left\{h_{\alpha}: \alpha \in c\right\}$ é linearmente independente. Suponhamos que $f \in \operatorname{hom}(G) \backslash<B>$; então $B \cup\{f\}$ é um conjunto linearmente independente, assim pela proposição 3.4.2, o subgrupo $f^{-1}(0)$ é denso em $G$, e o subconjunto $G \backslash f^{-1}(0)$ também é denso em $G$. Isto mostra que $f$ é um homomorfismo não contínuo de $G$ em 2 . O que contradiz a hipótese de $G$ ser um subgrupo pseudo-maximal de $2^{c}$. 
Teorema 3.4.11 Sejam $G, H$ dois subgrupos densos enumeráveis pseudo-maximais do cubo de Cantor de peso c. Existe um isomorfismo bicontínuo $\psi: 2^{c} \longrightarrow 2^{c}$ tal que $\psi[G]=H$.

Prova.Seja $G=<a_{n}: n \in \omega>$ e $H=<b_{n}: n \in \omega>$, e $\operatorname{seja} \varphi: G \longrightarrow H$ um isomorfismo algébrico tal que $\varphi\left(a_{n}\right)=b_{n}$. Então $\varphi$ é uma função bicontínua porque $G$ e $H$, têm a topologia de Bohr.

Com efeito, primeiro vamos abservar que se $h \in \operatorname{hom}(H)$ então existe um e somente um homomorfismo $g \in \operatorname{hom}(G)$ tal que $g=h \varphi$. Assim $\forall i \in 2$ e $\forall h \in \operatorname{hom}(H)$ temos $g^{-1}(i)=$ $\varphi^{-1}\left(h^{-1}(i)\right)$, e do lema 3.4.10 e da proposição 3.4 .4 vemos que $\varphi$ é uma função bicontínua. Então, da proposição 3.4 .9 , existe um isomorfismo bicontínuo $\psi: 2^{c} \longrightarrow 2^{c}$ tal que $\psi(x)=\varphi(x)$, $\forall x \in G$.

O próximo teorema, nos dá uma resposta à questão 1.1.3.

Observando que se $X$ é um subespaço denso e enumerável do cubo $2^{\kappa}$, então $\langle X\rangle$, é um subgrupo booleano, denso neste cubo, e contem $X$ como subespaço denso, é dificil imaginar uma resposta, à questão 1.1.3, com hipóteses mais fracas.

Teorema 3.4.12 Seja $(G,+, \tau)$ um grupo topológico booleano enumerável de peso $w(G)=\kappa$ onde $\aleph_{1} \leq \kappa \leq c$. Se $\tau \subset \tau_{\text {Bohr }}$ então existe um isomorfismo bicontinuo $\psi: G \longrightarrow H$, onde $H \subset 2^{\kappa}$, é um subgrupo topológico denso no cubo de Cantor de peso $\kappa$.

Prova. Por hipótese, $\tau \subset \tau_{B o h r}$ implica que existe um sistema fundamental de vizinhanças de $0 \in G$ da forma $\mathcal{B}(0)=$ $\left\{h_{\alpha}{ }^{-1}(0): \alpha \in \kappa\right\} \subset K H O M(G)$. Da proposição 3.4 .2 podemos 
obter uma família independente $\mathcal{A} \subset \mathcal{B}(0)$ com a propriedade maximal, isto é, $\left\{\cap \mathcal{A}_{0}: \mathcal{A}_{0} \in[\mathcal{A}]^{<\omega} \backslash\{\emptyset\}\right\}=\mathcal{B}(0)$. Consideremos agora a imersão canônica $\psi_{\mathcal{A}}=\psi$, do grupo $G$, que é definida por $\forall x \in G, \forall h \in \mathcal{A}, \psi(x)(h)=h(x)$. Seja $H=\psi[G] \subset 2^{\mathcal{A}}$ o subgrupo denso.

Vamos agora fazer mais uma observação a respeito deste problema. Os teoremas que obtivemos nesta seção, dependem da proposição 3.4.2. Vamos mostrar que esta proposição depende da estrutura algébrica dos grupos booleanos.

Nota 3.4.13 ( $\mathrm{CH})$

Seja $X$ um espaço regular, enumerável, denso em si mesmo, de peso $w(X)=c$, e pi-carácter local $\pi \chi(x, X)=c,(\forall x \in X)$.

Seja Cont $(X)$ o conjunto de todas as funçôes continuas de $X$ em 2, com a soma definida por $(f+g)(x)=f(x)+g(x), \forall x \in X$. Temos $\mathcal{D}_{0}(X)=\left\{f^{-1}(0): f \in \operatorname{Cont}(X)\right\}$, e $(\operatorname{Cont}(X),+)$ é um grupo booleano.

Seja $\mathcal{B} \subset \operatorname{Cont}(X)$ um conjunto linearmente independente, cujas funções do subgrupo $\langle\mathcal{B}\rangle$ separam pontos de fechados, portanto $\mathcal{A}=\left\{f^{-1}(0): f \in \mathcal{B}\right\}$ é subbase do espaço $X$. Vamos supor que $X$ é um espaço maximal. Pela proposição 2.1 de [ASTTW], o espaço $X$ não pode ser densamente imerso em $2^{\mathcal{A}}$, portanto, do lema 3.1.3, segue que $\mathcal{A}$ não é uma família independente. 


\section{Capítulo 4}

\section{Espaços homogêneos especiais}

\subsection{Topologias para $A u t(X)$}

Nesta seção, vamos estudar as topologias mais naturais, que podemos definir para o conjunto dos homeomorfismos de um espaço $X$ sobre si mesmo. Um dos problemas que motivaram este estudo, foi proposto em [Mill]: "caracterizar o produto topológico de $\omega$ cópias do espaço dos números racionais $\mathbb{Q}$ ".

Recentemente Alan Dow e Elliott Pearl estabeleceram o seguinte fato.

Fato 4.1.1 (DP) Se $X$ é um espaço de Hausdorff, zerodimensional, e $\chi(X)=\aleph_{0}$, então o espaço produto $X^{\aleph_{0}}$ é um espaço homogêneo.

Também este fato nos motivou a estudar quais as topologias que podemos definir no conjunto dos homeomorfismos de um espaço homogêneo não metrizável, e que resultassem em espaços com boas propriedades, preferivelmente, espaços já conhecidos!

Seja $(X, \tau)$ um espaço topológico com as seguintes propriedades: 
(1) X é um espaço de Hausdorff denso em si mesmo,

(2) $X$ é um espaço homogêneo,

(3) $X$ é um espaço separável ( existe um subconjunto denso e enumerável $A \subset X)$,

(4) $\forall x \in X, \chi(x, X)=\aleph_{0}$.

Definição 4.1.2 Denotamos por Aut $(X)$ o conjunto de todos os homeomorfismos de $X$ sobre $X$. Para cada $x \in X$ a função avaliação no ponto $x$ é definida por: $e_{x}: A u t(X) \longrightarrow X$ tal que $e_{x}(h)=h(x)$ para cada $h \in A u t(X)$.

Fato 4.1.3 A topologia de subespaço $A u t(X) \subset X^{X}$ é gerada pela família $\left\{e_{a}^{-1}(V): V \in \tau\right.$ e $\left.a \in X\right\}$.

Prova. Para todo $x \in X$ e todo $V \in \tau(X)$ temos $e_{x}{ }^{-1}(V)=$ $\operatorname{Aut}(X) \cap \pi_{x}{ }^{-1}(V)$.

Ainda vamos considerar alguns fatos gerais, que tem mais o sentido de fixar uma notação. Seja $A \subset X$ um subconjunto denso e enumerável.

Fato 4.1.4 A função $\psi_{A}: A u t(X) \longrightarrow{ }^{A} X$ definida por $\psi_{A}(h)(a)=h(a), \forall a \in A$ é injetora. Se damos a Aut $(X)$ a topologia cuja subbase é $\left\{e_{a}^{-1}(V): V \in \tau\right.$ e a $\left.\in A\right\}$, que denotamos por $\sigma_{A}$, então $\psi_{A}$ é um homeomorfismo sobre sua imagem $\psi_{A}[\operatorname{Aut}(X)]$.

Prova. Se $f, g \in A u t(X)$ são duas funções diferentes, existe $a \in$ $A$ tal que $f(a) \neq g(a)$, uma vez que $X$ é um espaço de Hausdorff, e $A$ é denso em $X$. Assim, $\psi_{A}(f)=\psi_{A}(g)$ implica $f=g$, o que mostra que $\psi_{A}$ é injetora. Como para todo $a \in A$ e todo 
$f \in \operatorname{Aut}(X)$ temos que vale $\psi_{A}(f)(a)=f(a)=\pi_{a}(f)=e_{a}(f)$, então $\psi_{A}$ é um homeomorfismo sobre sua imagem $\psi_{A}[$ Aut $(X)]$.

Fato 4.1.5 Nas condições acima, o espaço $\left(A u t(X), \sigma_{A}\right)$ satisfaz o primeiro axioma de enumerabilidade, isto é, $\chi(A u t(X))=$ $\aleph_{0}$. Se $X$ é um espaço de Tychonoff então o espaço $\left(\right.$ Aut $\left.(X), \sigma_{A}\right)$, também é um espaço de Tychonoff. Se o espaço produto $X^{A}$ é homeomorfo ao espaço $X$, e o espaço $X$ é hereditariamente separável, ( por exemplo, se ele é metrizável) então $\left(A u t(X), \sigma_{A}\right)$ também é separável.

Com efeito, como $A$ é enumerável, e $X$ satisfaz o primeiro axioma de enumerabilidade, o mesmo vale para o espaço produto $X^{A}$, o mesmo ainda vale para seus subespaços.

Vamos supor que o espaço $(X, \tau)$ é completamente metrizável, separável, zero-dimensional, e que $X^{\omega}$ é homeomorfo ao espaço $X$. Então fixado um subconjunto denso e enumerável $A \subset X$, podemos considerar $\left(A u t(X), \sigma_{A}\right)$ como um subespaço de $X$. Assim $A u t(X)$ como subespaço de $X$ é metrizável, separável e zero-dimensional.

Questão 4.1.6 $\psi_{A}[$ Aut $(X)]$ é um subespaço denso de $X^{A}$ ?

Vamos a seguir introduzir uma noção mais forte de espaços homogêneos,e que responde à questão acima proposta.

Definição 4.1.7 Dizemos que um espaço $X$ é finitamente homogêneo, se para todo $n \in \omega$ e toda função bijetora $j: F \longrightarrow G$, onde $|F|=n=|G|$ e $F, G \in[X]^{<\omega}$, existe $h \in A u t(X)$ tal que $h \uparrow F=j . \quad$ Em outros termos, toda bijeção entre subconjuntos finitos de $X$ estende-se a um auto-homeomorfismo de $X$. 
Na topologia que estamos definindo para $A u t(X)$, que denotamos por $\sigma_{A}$, somente usamos a topologia de $X$ e as funções avaliações nos pontos de $A$. Assim, podemos tomar esta definição para espaços $X$ de carácter não enumerável, e que satisfazem as demais hipóteses que consideramos.

A próxima proposição, também é válida num contexto um pouco mais geral, por exemplo, no cubo de Cantor $X=2^{\kappa}$ para um cardinal $\aleph_{1} \leq \kappa \leq c$.

Proposição 4.1.8 Suponhamos que $X$, além de todas as condiçôes já fixadas, (de 1 a 4 ), também é finitamente homogêneo. Então $\psi_{A}[$ Aut $(X)]$ é um subespaço denso de $X^{A}$.

Prova. Seja $\emptyset \neq W=\bigcap_{i<n} \pi_{a_{i}}{ }^{-1}\left(V_{i}\right)$ um aberto básico da topologia produto de $X^{A}$. Como cada um dos abertos $V_{i}$ é infinito, se temos $f \in W$ (portanto $f\left(a_{i}\right) \in V_{i}$ para cada $0 \leq i \leq n$ ) podemos escolher $\left\{b_{i} \in V_{i}: i \leq n\right\}=G$ tal que a função, $j: F \longrightarrow G$ definida por $j\left(a_{i}\right)=b_{i}$ seja uma bijeção de $F=\left\{a_{i}: i \leq n\right\}$ sobre $G$. Por hipótese, existe $h \in A u t(X)$ tal que $h\lceil F=j$. Mas então $\forall i \leq n, h\left(a_{i}\right) \in V_{i}$ ou seja ; $\psi_{A}(h) \in W \cap \psi_{A}[A u t(X)]$, o que mostra que $\psi_{A}[A u t(X)]$ é um subespaço denso de $X^{A}$.

Recordamos a definição de espaços densamente enumeravelmente homogêneos.

Definição 4.1.9 Um espaço topológico $X$ é densamente enumeravelmente homogêneo,se dados $A, B \subset X$ densos e enumeráveis, existe $\varphi \in A u t(X)$ tal que $\varphi[A]=B$.

Proposição 4.1.10 Suponhamos que $X$ seja densamente enumeravelmente homogêneo. A função definida por $\Psi(h)=\varphi \circ h \circ \varphi^{-1}$, para cada $h \in A u t(X)$, é um homeomorfismo de $\left(A u t(X), \sigma_{A}\right)$ sobre $\left(A u t(X), \sigma_{B}\right)$. 
Prova. Fixemos $a \in A, b \in B$ tais que $\varphi(a)=b$. Considerando as funções avaliações $e_{a}, e_{b}$ temos que $e_{b} \circ \Psi(h)=\varphi\left(h\left(\varphi^{-1}(b)\right)\right)=$ $\varphi \circ e_{a}(h)$ para todo $h \in A u t(X)$. Assim

$\Psi^{-1}\left(e_{b}{ }^{-1}(V)\right)=e_{a}^{-1}\left(\varphi^{-1}(V)\right)$. Assim efetivamente $\Psi$ é um homeomorfismo.

Seja $\mathbb{R}$ a reta real. Este é um exemplo de um espaço homogêneo, densamente homogêneo, mas que não é finitamente homogêneo. Para ver que esta afirmação é verdade, é suficiente considerar $F=\left\{-1,0, \frac{1}{2}\right\}, G\left\{1,0, \frac{1}{2}\right\}$ e $j(-1)=1, j(0)=$ $0, j\left(\frac{1}{2}\right)=\frac{1}{2}$, e não existe $h \in A u t(\mathbb{R})$ tal que $h\lceil F=j$.

Proposição 4.1.11 Seja $X$ um espaço homogêneo zero-dimensional cujos abertos-fechados não vazios, são, dois a dois, homeomorfos. Então $X$ é finitamente homogêneo.

Prova. Por indução em $n \in \omega$. Seja $J_{n}=\left\{\left(a_{i}, b_{i}\right): i \leq n\right\}$ tal que a função $j\left(a_{i}\right)=b_{i}: 0 \leq i \leq n$ é bijetora. Para $n=0$ e $J_{0}=\left\{\left(a_{0}, b_{0}\right)\right\}$ como o espaço $X$ é homogêneo temos que existe $h \in \operatorname{Aut}(X)$ tal que $h\left(a_{0}\right)=b_{0}$. Sejam $\mathcal{A}=\left\{U_{j}: j \leq n\right\} \mathrm{e}$ $\mathcal{B}=\left\{V_{j}: j \leq n\right\}$ recobrimentos celulares de $X$ tais que $\forall j \leq n$ valha exatamente $J_{n} \cap U_{j} \times V_{j}=\left\{\left(a_{j}, b_{j}\right)\right\}$. Como cada um dos abertos-fechados, acima, envolvidos na construção, é uma cópia de $X$, temos os homeomorfismos, $h_{j}: U_{j} \longrightarrow V_{j}$ tal que $h_{j}\left(a_{j}\right)=b_{j}$ para cada $j \leq n$. Seja $h=\bigcup_{j \leq n} h_{j}$ o homeomorfismo resultante. Temos que $h\left(a_{j}\right)=h_{j}\left(a_{j}\right)=\bar{b}_{j}$.

\subsection{Métricas completas para $\operatorname{Aut}(X)$}

Vamos supor que $(X, \rho)$ é um espaço métrico completo separável, zerodimensional, homogêneo, que satisfaz $X^{\aleph_{0}} \approx X$. Vamos 
definir uma métrica para $A u t(X)$ que faz deste conjunto um espaço métrico completo, e cuja topologia refina a topologia $\sigma_{A}$ que introduzimos na seção anterior. Estamos interessados na caracterização de $A u t(X)$, com estas "novas topologias", quando $X=2^{\omega}$ e $X=B(\kappa)$ para $\aleph_{0} \leq \kappa \leq c .(\operatorname{pg} 8)$

Definição 4.2.1 Seja $(X, d)$ um espaço métrico completo, onde $d(x, y) \leq 1(\forall x, y \in X)$. Denotamos por $(C(X, X), \rho)$ o espaço métrico das funções contínuas de $X$ em $X$, onde a métrica é definida por:

$$
\rho(f, g)=\sup \{d(f(x), g(x)): x \in X\} .
$$

Usamos a notação $\rho$-sequência de Cauchy para indicar as sequências de Cauchy, do espaço $(C(X, X), \rho)$.

Fato 4.2.2 Se $\left\{f_{n}\right\}_{n \in \omega}$ é uma $\rho$-sequência de Cauchy, então $(\forall x \in X)\left\{f_{n}(x)\right\}_{n \in \omega}$ é uma sequência de Cauchy em $X$.

Assim os limites de sequências no espaço das funções, como estamos considerando, é o limite uniforme, portanto também temos o fato abaixo enunciado.

Fato 4.2.3 Se $\left\{f_{n}\right\}_{n \in \omega}$ é uma $\rho$-sequência de Cauchy, então a função $f: X \longrightarrow X$, tal que

$(\forall x \in X)\left(f(x)=\lim _{n \in \omega} f_{n}(x)\right)$ pertence a $C(X, X) ;$ $e(C(X, X), \rho)$ é um espaço métrico completo.

Vamos considerar $(A u t(X), \varrho)$ onde a nova métrica é a modificação necessária da métrica de subespaço de $A u t(X) \subset C(X, X)$, para fazê-lo um espaço métrico completo. 
Definição 4.2.4 Sejam $f, g \in A u t(X)$. Definimos

$$
\varrho(f, g)=\max \left\{\rho(f, g), \rho\left(f^{-1}, g^{-1}\right)\right\} .
$$

Seja $(\operatorname{Aut}(X), \varrho)$ o espaço métrico resultante.

É imediato que $\varrho(f, g)=\varrho\left(f^{-1}, g^{-1}\right)$. Também $\rho(f, g) \leq$ $\varrho(f, g)$ e $\rho\left(f^{-1}, g^{-1}\right) \leq \varrho\left(f^{-1}, g^{-1}\right)$.

Usamos a notação $\varrho$-sequência de Cauchy para indicar as sequências de Cauchy do espaço $(A u t(X), \varrho)$. O fato abaixo é uma generalização do mesmo fato em [AO], para espaços métricos não localmente compactos.

Fato 4.2.5 (Aut $(X), \varrho)$ é um espaço métrico completo.

Prova.Toda $\varrho$-sequência de Cauchy $\left\{f_{n}\right\}_{n \in \omega}$ determina duas $\rho$ sequências de Cauchy, e seus respectivos limites:

$$
\lim _{\rho} f_{n}=g, \lim _{\rho} f_{n}^{-1}=h \in C(X, X) .
$$

Ainda as duplas sequências $\left\{f_{n}{ }^{-1} \circ f_{m}\right\}_{n, m \in \omega}$ e $\left\{f_{m}{ }^{-1} \circ f_{n}\right\}_{n, m \in \omega}$ convergem para a identidade $i d: X \longrightarrow X$, em $C(X, X)$. Fixado $x \in X$, dado $\epsilon>0$ existe $\delta>0$ e para este $\delta<\epsilon$ existe $n_{0}$ tal que $n \geq n_{0}$ implica $\max \left\{d\left(f_{n}(y), g(y)\right), d\left(f_{n}^{-1}(y), h(y)\right)\right\} \leq \delta<$ $\epsilon$, para todo $y \in X$. Mas então, $d(x, g(h(x)))<\epsilon$, o que mostra que $h^{-1}=g$.

Supomos que $(X, d)$ é um espaço métrico completo, homogêneo, separável, com $\operatorname{Ind}(X)=0$ e que $d$ é uma ultramétrica de $X$. Logo abaixo, na próxima seção, relembramos a definição de ultramétrica.

Vamos descrever a relação que existem entre as topologias que introduzimos acima para o conjunto $A u t(X)$. Primeiro, $\sigma_{A}$, 
é menos fina que $\tau_{\varrho}$ que é a topologia induzida pela métrica $\varrho$. Com efeito, dados $a \in A$ e $V \in \tau$ tal que existe um homeomorfismo $h \in \operatorname{Aut}(X)$, e $e_{a}(h)=h(a) \in V$, então existe $\epsilon>0$ tal que $B_{d}(h(a), \epsilon) \subset V$, portanto $B_{\varrho}(h, \epsilon) \subset e_{a}^{-1}(V)$, pois pela definição desta métrica, se $f \in B_{\varrho}(h, \epsilon)$ então $d(f(a), h(a)) \leq \epsilon$. Seja, Inv $: h \in \operatorname{Aut}(X) \longrightarrow h^{-1} \in \operatorname{Aut}(X)$ a inversão de homeomorfismos em $A u t(X)$. Para cada $f \in A u t(X)$ e cada $\epsilon>0$ definimos $W(f, \epsilon)=\bigcap_{a \in A}\left[e_{a}^{-1}\left(B_{d}(f(a), \epsilon)\right) \cap e_{a}^{-1}\left(B_{d}\left(f^{-1}(a), \epsilon\right)\right)\right]$. Como $A \subset X$ é denso em $X$, e temos que

$$
\begin{aligned}
h \in W(f, \epsilon) & \Longleftrightarrow(\forall a \in A)\left(h(a) \in B_{d}(f(a), \epsilon) \cap B_{d}\left(f^{-1}(a), \epsilon\right)\right) \\
& \Longleftrightarrow h \in B_{\varrho}(f, \epsilon), \\
\text { então, } W(f, \epsilon) & =B_{\varrho}(f, \epsilon) .
\end{aligned}
$$

Assim, temos uma idéia de como tentar generalizar alguns fatos para os espaços não metrizáveis. Por exemplo $A u t\left(2^{\kappa}\right)$ para o cardinal $\aleph_{1} \leq \kappa \leq c$. Observando os fatos acima, pareceu-nos que devíamos procurar uma estrutura uniforme, que faria da inversão Inv, um isomorfismo desta estrutura. Nas condições acima, Inv é uma isometria.

\subsection{Ultramétricas}

Nesta seção vamos citar os fatos relevantes, para as nossas aplicações, a respeito dos espaços metrizáveis, $X$, com a grande dimensão indutiva igual a zero, $\operatorname{Ind}(X)=0$.

Vamos relembrar a definição das ultramétricas. Uma métrica $d: X \times X \longrightarrow[0,1]$ é uma ultramétrica ou métrica não arquimediana, se $\forall x, y, z \in X$ a desigualdade triangular tem a forma: $d(x, z) \leq \max \{d(x, y), d(y, z)\}$. 
Se $d$ é uma ultramétrica, então as bolas abertas $B_{d}(x, \epsilon)$, para todos os pontos $x \in X$ e todo $\epsilon>0$, são abertos-fechados na topologia induzida por esta métrica.

Fato 4.3.1 (Hausdorff, 1934)[Eng] Seja X um espaço metrizável. São equivalentes as seguintes afirmaçôes:

(1) $\operatorname{Ind}(X)=0$

(2) a topologia de $X$ admite uma ultramétrica

$d: X \times X \longrightarrow[0,1]$.

Fato 4.3.2 Seja $C=\{0,1\}^{\aleph_{0}}$ o conjunto de Cantor. Uma ultramétrica compatível com a sua topologia é dada por

$d(s, t)=(1+\min \{n: s(n) \neq t(n)\})^{-1}$ se $s \neq t$ e $s, t \in 2^{\omega}$. (pg 8)

Fato 4.3.3 Seja $P=N^{\aleph_{0}}$ o conjunto de Baire, isto é, o espaço dos números irracionais. Uma ultramétrica compativel com a sua topologia é dada por

$$
d(s, t)=(1+\min \{n: s(n) \neq t(n)\})^{-1} \text { se } s \neq t \text { e } s, t \in \omega^{\omega} .
$$
(pg 8)

Fato 4.3.4 Seja $B(\kappa)=D(\kappa)^{\aleph_{0}}$ o espaço de Baire, isto é, o espaço produto de $\aleph_{0}$ cópias do espaço discreto $D(\kappa)=\kappa$. Uma ultramétrica compativel com a sua topologia é dada por $d(s, t)=(1+\min \{n: s(n) \neq t(n)\})^{-1}$ se $s \neq t$ e $s, t \in \kappa^{\omega}$. (pg 8)

\section{$4.4(A u t(P), \varrho)$ é homeomorfo a $B(c)$}

Nesta seção vamos mostrar que $(A u t(C), \varrho) \approx P,(A u t(P), \varrho) \approx$ $B\left(2^{\aleph_{0}}\right)$, e fica claro que $(A u t(B(\kappa)), \varrho) \approx B\left(2^{\kappa}\right)$. 
Teorema 4.4.1 Seja $P=\left(N^{\aleph_{0}}, d\right)$ o espaço métrico de Baire de peso $\aleph_{0}$. Então $($ Aut $(P), \varrho)$ é homeomorfo a $B\left(2^{\aleph_{0}}\right)$

Prova. Do fato 4.3.3, $H(P)=(A u t(P), \varrho)$ é um espaço métrico completo, e $\varrho$ é uma ultramétrica. Precisamos mostrar que $H(P)$ é homogêneo em peso, $(\mathrm{pg} 8)$ de peso $w(H(P))=c$. Vamos tomar um recobrimento celular infinito de $P \times P$,

$\mathcal{B}_{n}=\left\{U_{n} \times V_{n}: n \in \omega\right\}$

cujos elementos são as bolas na métrica produto, $B_{d}\left(x, \frac{1}{n+1}\right) \times B_{d}\left(y, \frac{1}{n+1}\right) \subset P \times P,(\forall x, y \in P)$.

Para cada $n \in \omega$ podemos escolher um homeomorfismo $h_{n, m}$ : $U_{n} \longrightarrow V_{m}$, e denotamos por graf $\left(h_{n, m}\right)$ o seu gráfico, e para cada permutação $\sigma \in S_{\omega} \subset{ }^{\omega} \omega$ tomamos $\bigcup_{n \in \omega} \operatorname{graf}\left(h_{n, \sigma(n)}\right)=$ graf $\left(h_{\sigma}\right)$, que é o gráfico de um elemento $h_{\sigma} \in H(P)$. Dados $\sigma_{1} \neq \sigma_{2} \in S_{\omega}$ temos $\varrho\left(h_{\sigma_{1}}, h_{\sigma_{2}}\right) \geq \frac{1}{n+1}$ o que mostra-nos que $\left\{h_{\sigma}: \sigma \in S_{\omega}\right\}$ é fechado e discreto em $H(P)$. Assim $w(H(P))=$ $e(H(P))=\left|S_{\omega}\right|=c$, onde $e(H(P))$ é o extent deste espaço.(pg 4)

Agora, precisamos mostrar que $H(P)$ é homogêneo em peso. Dado $h \in H(P)$ e uma vizinhança $h \in B_{\varrho}\left(h, \frac{1}{n+1}\right)$ deste ponto, fixamos os elementos do recobrimento celular $\left\{B_{d}\left(x, \frac{1}{n+1}\right) \times\right.$ $\left.B_{d}\left(h(x), \frac{1}{n+1}\right): x \in P\right\}$ do gráfico deste homeomorfismo, $h$, escolhemos uma desta vizinhanças $\Omega=B_{d}\left(x, \frac{1}{n+1}\right) \times B_{d}\left(h(x), \frac{1}{n+1}\right)$, e como no parágrafo anterior definimos uma família discreta e fechada de homeomorfismos, cujos os gráficos diferem de graf $(h)$ somente nesta vizinhança $\Omega$, mas nela estando contidos.

Assim, $w\left(B_{\varrho}\left(h, \frac{1}{n+1}\right)\right)=c$. Do teorema de A. H. Stone, $(\mathrm{pg} 8)$ segue que $(A u t(P), \varrho) \approx B(c)$.

Uma demonstração totalmente análoga vale para o seguinte teorema. 
Teorema 4.4.2 Seja $B(\kappa)=\left(D(\kappa)^{\aleph_{0}}, d\right)$ o espaço métrico de Baire de peso $\kappa$. Então

$(A u t(B(\kappa)), \varrho)$ é homeomorfo a $B\left(2^{\kappa}\right)$

Prova. Do fato 4.3.4, $H(B(\kappa))=(A u t(B(\kappa)), \varrho)$ é um espaço métrico completo, e $\varrho$ é uma ultramétrica. Precisamos mostrar que $H(B(\kappa))$ é homogêneo em peso, (pg 8) de peso $w(H(P))=$ $2^{\kappa}$. Vamos tomar um recobrimento celular infinito de $B(\kappa) \times$ $B(\kappa), \mathcal{B}_{n}=\left\{U_{n} \times V_{n}: n \in \omega\right\}$ cujos elementos são as bolas na métrica produto,

$B_{d}\left(x, \frac{1}{n+1}\right) \times B_{d}\left(y, \frac{1}{n+1}\right) \subset B(\kappa) \times B(\kappa),(\forall x, y \in B(\kappa))$.

Para cada $\alpha \in \kappa$ podemos escolher um homeomorfismo $h_{\alpha, \beta}$ : $U_{\alpha} \longrightarrow V_{\beta}$, e denotamos por graf $\left(h_{\alpha, \beta}\right)$ o seu gráfico, e para cada permutação $\sigma \in S_{\kappa} \subset{ }^{\kappa} \kappa$ tomamos $\bigcup_{\alpha \in \kappa} \operatorname{graf}\left(h_{\alpha, \sigma(\alpha)}\right)=$ graf $\left(h_{\sigma}\right)$, que é o gráfico de um elemento $h_{\sigma} \in H(B(\kappa))$. Dados $\sigma_{1} \neq \sigma_{2} \in S_{\kappa}$ temos $\varrho\left(h_{\sigma_{1}}, h_{\sigma_{2}}\right) \geq \frac{1}{n+1}$ o que mostra-nos que $\left\{h_{\sigma}\right.$ : $\left.\sigma \in S_{\omega}\right\}$ é fechado e discreto em $H(B(\kappa))$. Assim $w(H(B(\kappa)))=$ $e(H(B(\kappa)))=\left|S_{\omega}\right|=2^{\kappa}$, onde $e(H(B(\kappa)))$ é o extent deste espaço.(pg 8).

Agora, precisamos mostrar que $H(B(\kappa))$ é homogêneo em peso. Dado $h \in H(B(\kappa))$ e uma vizinhança $h \in B_{\varrho}\left(h, \frac{1}{n+1}\right)$ deste ponto, fixamos os elementos do recobrimento celular $\left\{B_{d}\left(x, \frac{1}{n+1}\right) \times\right.$ $\left.B_{d}\left(h(x), \frac{1}{n+1}\right): x \in B(\kappa)\right\}$ do gráfico deste homeomorfismo, $h$, escolhemos uma desta vizinhanças $\Omega=B_{d}\left(x, \frac{1}{n+1}\right) \times B_{d}\left(h(x), \frac{1}{n+1}\right)$, e como no parágrafo anterior definimos uma família discreta e fechada de homeomorfismos, cujos os gráficos diferem de graf $(h)$ somente nesta vizinhança $\Omega$, mas nela estando contidos.

Assim, $w\left(B_{\varrho}\left(h, \frac{1}{n+1}\right)\right)=2^{\kappa}$. Do teorema de A. H. Stone, $(\mathrm{pg}$ 8) segue que $(A u t(B(\kappa)), \varrho) \approx B\left(2^{\kappa}\right)$.

Teorema 4.4.3 Seja $C=\left(2^{\aleph_{0}}, d\right)$ o cubo de Cantor de peso $\aleph_{0}$. Então 
$($ Aut $(C), \varrho)$ é homeomorfo a $B\left(\aleph_{0}\right)=P$

Prova. Como o conjunto de Cantor $C$ é compacto, em [AO] prova-se que o espaço métrico completo $(A u t(C), \varrho)$ é um grupo topológico separável, para a composição de homeomorfismos, não compacto. Assim, nenhum aberto-fechado, exceto o vazio, é compacto. Do teorema de Urysohn e Alexandroff, segue-se $(\operatorname{Aut}(C), \varrho) \approx P$.

\subsection{Para espaços uniformes}

Nesta seção, vamos estudar uma generalização de alguns dos fatos que obtivemos nas seções precedentes. Para tal fim, vamos necessitar da noção de espaços uniformes, e recordamos logo abaixo as principais definições e proposições a respeito deste tema.

\subsection{1 (Kelley, pg 65)}

Uma ordem parcial $\geq$ dirige um conjunto $T$, se $T$ é não vazio, e se $s$ e $t$ são membros de $T$, então existe $r \in T$ tal que $r \geq s, r \geq t$. O par $(T, \geq)$ é chamado conjunto dirigido, e como é usual, o representamos por $T$.

Uma rede (net), $\left\{x_{t}: t \in T\right\}$, é uma função de um conjunto dirigido $T$ em um conjunto não vazio $X$.

Suponhamos que $X$ é um espaço de Hausdorff. Dizemos que a rede $\left\{x_{t}: t \in T\right\}$ converge para um ponto $x \in X$, e escrevemos $x_{t} \longrightarrow x$ ou ainda $\lim x_{t}=x$, se para toda vizinhança aberta $V \subset X$ do ponto $x$ em $X$, existe $t_{V} \in T$ tal que $t \geq t_{V}$ implica $x_{t} \in V$. É usual dizer a rede $\left\{x_{t}: t \in T\right\}$ está eventualmente contida em $V$. Uma rede $\left\{x_{s}: s \in S\right\}$, é uma sub-rede da rede $\left\{x_{t}: t \in T\right\}$ se $S \subset T$, e $(S, \geq)$ é cofinal em $(T, \geq)$. 
Vamos relembrar a definição de uniformidade e de espaços uniformes, e também alguns fatos básicos desta teoria.

\subsection{2 (Kelley, pg 176)}

Seja $X$ um conjunto não vazio. A diagonal do quadrado $X \times X$ é o subconjunto: $\Delta(X)=\{(x, x): x \in X\}$. Uma uniformidade para o conjunto $X$, é uma família não vazia $\mu$ de subconjuntos de $X \times X$, tais que

(1) cada membro de $\mu$ contém a diagonal $\Delta(X)$,

(2) se $U \in \mu$ então $U^{-1}=\{(y, x):(x, y) \in U\} \in \mu$,

(3) se $U \in \mu$ então existe $V \in \mu$ tal que $V \circ V \subset U$,

(4) se $U$ e $V$ são membros de $\mu$, então $U \cap V \in \mu$ e

(5) se $U \in \mu$ e $U \subset V \subset X \times X$, então $V \in \mu$.

Ao par $(X, \mu)$ chamamos de espaço uniforme. A topologia $\tau_{\mu}$ induzida pela uniformidade $\mu$, é a família de todos os subconjuntos $A \subset X$ tais que $(\forall x \in A)(\exists U \in \mu)$ tal que $U[x]=$ $\{y \in X:(x, y) \in U\} \subset A$. Denotamos o espaço topológico correspondente por $\left(X, \tau_{\mu}\right)$.

Dizemos que uma função $f$ de um espaço uniforme $(X, \mu) \mathrm{em}$ um espaço uniforme $(Y, \nu)$ é uniformemente contínua se e somente se $(\forall V \in \nu)(\{(a, b) \in X \times X:(f(a), f(b)) \in V\} \subset \mu$. Se a função $f$ é uniformemente contínua, então ela é contínua em relação as topologias induzidas pelas respectivas uniformidades. Se a função $f$ é bijetora, e ambas $f$ e $f^{-1}$ são uniformemente contínuas, então dizemos que $f$ é um isomorfismo uniforme.

Uma subfamília $\mathcal{B}$, é uma base da uniformidade $\mu$ se e somente se cada membro de $\mu$ contém um membro de $\mathcal{B}$. Uma subfamília $\mathcal{A}$, é uma subbase (ou conjunto gerador) da uniformidade $\mu$ se e somente se a família das interseções de finitos membros de $\mathcal{A}$, formam uma base de $\mu$. 
Um calibre (gage) P para um espaço uniforme $(X, \mu)$ é a família de todas as pseudo-métricas $d: X \times X \longrightarrow \mathbb{R}$ que são uniformemente continuas em $X \times X$.

\subsection{3 (Kelley, pg 190)}

Uma rede $\left\{x_{t}: t \in T\right\}$ em um espaço uniforme $(X, \mu)$ é uma rede de Cauchy, escrevemos rede $\mu$-Cauchy, se e somente se $(\forall U \in \mu)\left(\exists t_{U} \in T\right)\left(s, t \geq t_{U} \Longrightarrow\left(x_{s}, x_{t}\right) \in U\right)$.

Um espaço uniforme $(X, \mu)$ é uniformemente completo se toda rede $\mu$-Cauchy converge em $\left(X, \tau_{\mu}\right)$.

\subsection{4 (Kelley, pg 226)}

Seja $F$ a família de todas as funções de um conjunto $X$ em um espaço uniforme $(Y, \nu)$; e seja $\mu$ a uniformidade da convergência uniforme. Então:

(a) A uniformidade $\mu$ é gerada pela família de todas as pseudométricas da forma:

$$
\rho(f, g)=\sup \{d(f(x), g(x)): x \in X\}
$$

onde dé um elemento limitado do calibre de $(Y, \nu)$.

(b) Uma rede $\left\{f_{t}: t \in T\right\}$ em $F$ converge uniformemente para $g$ se e somente se $\left\{f_{t}(x): t \in T\right\}$ é uma rede $\nu$-Cauchy $e$ $f_{t}(x) \longrightarrow g(x)$ para cada $x \in X$.

(c) $S e(Y, \nu)$ é completo, então $(X, \mu)$ é completo.

Fixamos o espaço topológico produto

$$
X=\left(C, \tau_{d}\right)^{\kappa}
$$

para um cardinal não enumerável, $\kappa \leq c$, onde cada espaço $(C, d)$ é um espaço métrico completo, zero-dimensional, separável, com ultramétrica $d$, limitada em $[0,1]$. Como $\kappa \leq c$, o espaço $X$ é separável. 
Supomos também que $C$ é fortemente homogêneo; isto é; todos os abertos-fechados, exceto o vazio, são dois a dois homeomorfos.

Portanto, os abertos-fechados básicos do espaço produto são homeomorfos ao próprio espaço produto, e assim estes também são finitamente homogêneos.

Usamos, a notação $\pi_{\alpha}$ para as projeções nos espaços produtos, $\pi_{\alpha}(x)=x(\alpha)$, para cada $x \in X$.

Fixamos um subconjunto denso e enumerável, $A \subset X$, e para cada $a \in A$ seja $e_{a}: A u t(X) \longrightarrow X$, a avaliação no ponto $a \in A$, e para cada $\alpha \in \kappa$ e cada $f \in A u t(X)$, para cada $\epsilon, 0<\epsilon<1$, em relação à ultramétrica, que estamos considerando, definimos:

$W(a, f, \alpha, \epsilon)=e_{a}^{-1}\left(\pi_{\alpha}^{-1}\left(B_{d}(f(a)(\alpha), \epsilon)\right)=\left\{h \in A u t\left(C^{\kappa}\right):\right.\right.$ $d(h(a)(\alpha), f(a)(\alpha)) \leq \epsilon\}$.

Seja o espaço topológico $\left(A u t\left(C^{\kappa}\right), \sigma_{A}\right)$ cuja topologia têm por subbase a família:

$$
\mathcal{B}=\left\{W(a, f, \alpha, \epsilon): a \in A, f \in \operatorname{Aut}\left(C^{\kappa}\right), \alpha \in \kappa, \epsilon>0\right\}
$$

Esta definição é tomada de modo que valha o fato 4.1.3. Também $C^{\kappa}$ é finitamente homogêneo, e assim vale a proposição 4.1.7. Vamos reenunciar abaixo estas duas afirmações:

Fato 4.5.5 A função $\psi:$ Aut $\left(C^{\kappa}\right) \longrightarrow\left(C^{\kappa}\right)^{A}$ definida por $\psi(h)(a)=$ $h(a)$ é injetora. Se damos a $A u t\left(C^{\kappa}\right)$ a topologia $\sigma_{A}$, então $\psi$ é um homeomorfismo sobre sua imagem $\psi\left[\right.$ Aut $\left.\left(C^{\kappa}\right)\right]$, e esta por sua vez, é um subespaço denso em $\left(C^{\kappa}\right)^{A}$.

Vamos definir a seguir a topologia $\tau$ que refina $\sigma_{A}$, e estabelece $\left(A u t\left(C^{\kappa}\right), \tau\right)$, que de modo análogo aos dos espaços métricos que estudamos, $\left(A u t\left(C^{\kappa}\right), \tau\right)$ resulte em um espaço uniformemente completo. Para destaque, vamos usar a notação $\mathbb{C}=(C, d)$. 
Definição 4.5.6 Para cada $f \in A u t\left(\mathbb{C}^{\kappa}\right), \alpha \in \kappa e \epsilon>0$ seja o subconjunto de Aut $\left(\mathbb{C}^{\kappa}\right)$, definido por

$W(f, \alpha, \epsilon)=\bigcap_{a \in A}\left[\left\{h \in \operatorname{Aut}\left(\mathbb{C}^{\kappa}\right): d(h(a)(\alpha), f(a)(\alpha)) \leq \epsilon e\right.\right.$ $\left.\left.d\left(h^{-1}(a)(\alpha), f^{-1}(a)(\alpha)\right) \leq \epsilon\right\}\right]$.

Seja $\tau$ a topologia, para Aut $\left(\mathbb{C}^{\kappa}\right)$, cuja subbase de abertos é:

$\mathcal{G}=\left\{W(f, \alpha, \epsilon): f \in \operatorname{Aut}\left(\mathbb{C}^{\kappa}\right), \alpha \in \kappa e \epsilon>0\right\}$, e seja $\left(\right.$ Aut $\left.\left(\mathbb{C}^{\kappa}\right), \tau\right)$, o espaço topológico resultante.

Vamos a seguir definir os espaços uniformes $\left(\mathbb{C}^{\kappa}, \mu\right) \mathrm{e}$ $\left(\right.$ Aut $\left.\left(\mathbb{C}^{\kappa}\right), \nu\right)$, cujas topologias induzidas pelas uniformidades, são às topologias neles já definidas, e que resultam em espaços uniformemente completos.

Definição 4.5.7 Para cada $\alpha \in \kappa$ indicamos por $d_{\alpha}$ a mesma ultramétrica de $\mathbb{C}$, que temos usado neste texto. Seja $\mathcal{D}=\left\{d_{\alpha}\right.$ : $\alpha \in \kappa\}$ a familia resultante. Seja $\left(\mathbb{C}^{\kappa}, \mu\right)$ o espaço uniforme cuja uniformidade $\mu$ é induzida pela família de pseudo-métricas $\mathcal{D}$.

Para cada $\alpha \in \kappa$ indicamos por $\varrho_{\alpha}$ cada pseudo-ultramétrica sobre o conjunto $A u t\left(\mathbb{C}^{\kappa}\right)$, que são definidas por:

$$
\begin{aligned}
& \varrho_{\alpha}(f, g)=\max \left\{\rho_{\alpha}(f, g), \rho_{\alpha}\left(f^{-1}, g^{-1}\right)\right\} \text { onde } \\
& \left.\rho_{\alpha}(f, g)=\sup \left\{d_{\alpha}(f(x)(\alpha), g(x)(\alpha)): x \in \mathbb{C}^{\kappa}\right)\right\} .
\end{aligned}
$$

Seja $\mathcal{E}=\left\{\varrho_{\alpha}: \alpha \in \kappa\right\}$ a família resultante. Seja $\left(\right.$ Aut $\left.\left(\mathbb{C}^{\kappa}\right), \nu\right)$ o espaço uniforme cuja uniformidade $\nu$ é induzida pela familia de pseudo-métricas $\mathcal{E}$.

Fato 4.5.8 Para cada $\left.f \in A u t\left(\mathbb{C}^{\kappa}\right)\right), \alpha \in \kappa, \epsilon>0$ temos:

$W(f, \alpha, \epsilon)=B_{\varrho_{\alpha}}(f, \epsilon)$.

Portanto, a topologia $\tau$ é induzida pela uniformidade $\nu$. 
Teorema 4.5.9 $\left(\right.$ Aut $\left.\left(\mathbb{C}^{\kappa}\right), \nu\right)$ é um espaço uniformemente completo.

Prova. Seja $\left\{f_{t}: t \in \Gamma\right\}=S$ uma rede de Cauchy no espaço uniforme $\left(A u t\left(\mathbb{C}^{\kappa}\right), \nu\right)$. Para cada uma das pseudo-métricas $\varrho_{\alpha}$, a rede $\left\{\varrho_{\alpha}\left(f_{t}, f_{s}\right): t, s \in \Gamma \times \Gamma\right\}$ converge para o zero. Assim, fixados $x \in \mathbb{C}^{\kappa}$ e $\alpha \in \kappa$, temos $g_{\alpha}(x)=\lim _{t \in \Gamma} f_{t}(x)(\alpha)$ e $h_{\alpha}(x)=$ $\lim _{t \in \Gamma} f_{t}^{-1}(x)(\alpha)$, e então podemos definir as duas funções $g, h$ de $\mathbb{C}^{\kappa}$ em si mesmo, como limites uniformes $\left(g=\lim _{t \in \Gamma} f\right.$ e $\left.h=\lim _{t \in \Gamma} f_{t}^{-1}\right)$.

Por outro lado, a rede $\left\{f_{t} \circ f_{s}^{-1}:(t, s) \in \Gamma \times \Gamma\right\}=N$ converge para a identidade $i d \in A u t\left(\mathbb{C}^{\kappa}\right)$, e portanto, temos $\forall x \in \mathbb{C}^{\kappa}$ :

$$
\begin{aligned}
i d(x) & =\lim _{t, s} f_{t} \circ f_{s}^{-1}(x) \\
& =\lim _{t} \lim _{s} f_{t}\left(f_{s}^{-1}(x)\right) \\
& =\lim _{t} f_{t}\left(\lim _{s} f_{s}^{-1}(x)\right) \\
& =\lim _{t} f_{t}(h(x)) \\
& =g \circ h(x) .
\end{aligned}
$$

Analogamente, $h \circ g=i d$. Assim, mostramos que $\left(A u t\left(\mathbb{C}^{\kappa}\right), \nu\right)$ é uniformemente completo. 


\section{Referências Bibliográficas}

[AO] J. M. Arts, L. G. Oversteegen, The product structure of homogeneous spaces, Indag Mathem, (1990) 1-5.

[ASTTW] O. T. Alas, M. Sanchis, M. G. Tkačenko, V. V. Tkachuk, R. G. Wilson, Irresolvable and submaximal spaces: homogeneity vs $\sigma$-discreteness and new $Z F C$ examples, pre-print, 1999.

[BK] M. Bell, K. Kunen, On the Pi-character of Ultrafilters, C. R. Math. Rep. Acad. Sci. Canada 3(1981), 351-356.

[Co] W. W. Comfort, Topological Groups, The Handbook of settheoretic topology (K. Kunen and J Vaughan, eds) North Holland, 1984, 1143-1263.

[Eng] R. Engelking, General Topology, Heldermann Verlag, 1989.

[DP] A.Dow, E.Pearl, Homogeneity in powers of zero-dimensional first countable spaces,Proc. Amer. Math. Soc.125(1997)n0 8, 2503-2510. 
[Fr] Z. Frolik, Homogeneity problem for extremally disconected spaces, Comment. Math. Univ. Carolinae, 8, 4(1967), 757763.

[He] E. Hewitt, A problem of set-theoretic topology, Duke Math. J., 10(1943), 309-333.

[Juh] I. Juhász, Cardinal Function in Topology, Ten Years Later, Mathematish Centum Amsterdan, 1980.

[Kel] J. E. Kelley, General Topology, D. van Nostrand Company, Inc., 1961.

[Kun] K. Kunen, Set Theory: An introduction to independence proof, North-Holland, Amsterdan, 1980.

[KT] K. Kunen, F. D. Tall, On the consistency of the non-existence of Baire irresolvable space, pre-print.

[Ma] V. I. Malyhin,Irresolvable countable spaces of weight less than c, Comment. Math. Univ. Carolinae 40, 1(1999), 181-185.

[Med] S. V. Medvedev, Topological Characteristics of Spaces $Q(\kappa)$ and $Q \times B(\kappa)$, Vestinik Moskowskogo Universiteta Mathematika, 41, 1(1986), 42-45.

[Mill] Jan van Mill, Characterization of Some Zerodimensional Separable Metric Spaces, Trans. AMS 264, 1,(1981), 205-215. 
[Vau] J.E. Vaughan,Small uncountable cardinls and topology, Open Problems in Topology 195-216 (J. van Mill, G.M. Reed, eds) North Holland, 1984, Amsterdan, 1990. 